\title{
An effective XFEM with equivalent eigenstrain for stress intensity factors of homogeneous plates
}

\author{
Elena Benvenuti \\ Engineering Department, University of Ferrara, via Saragat, 1 Ferrara, 44122 Ferrara Italy, \\ elena.benvenuti@unife.it
}

\begin{abstract}
Based on the concept of equivalent eigenstrain, a low-order accurate eXtended Finite Element Method (XFEM) is presented. The aim is the determination of the stress intensity factors of cracked homogeneous specimens. The proposed approach differs from the conspicuous amount of existing contributions on this topic. The findings of the present paper highlight aspects so far neglected in the literature, such as the mechanical meaning of additional fields and equations specific to the XFEM approximation of the displacement field. Moreover, based on the plane strain examples simulated in the present study, the proposed XFEM is generally computationally more robust and accurate than existing comparable XFEMs, while keeping a minimal implementation effort.
\end{abstract}

Keywords: Stress intensity factor, XFEM, elasticity

\section{1. Introduction}

The present study proposes an eXtended Finite Element Method (XFEM) where the crack tips are modeled as Eshelby's elastic singularities. The target is to devise an effective model with minimal computational effort. With respect to comparable XFEMs for fracture, the stress-intensity factors are more accurate, while the numerical procedure is more robust.

The XFEM 1, 2, 3, 4, is a generalized Partition of Unity Finite Element Method (PUFEM) [5]. The Generalized Finite Element Method (GFEM) 6] is another PUFEM, structurally similar to the XFEM. Both GFEM and XFEM exploit the knowledge of the expected shape of the stress and the strain fields; the displacement field is indeed approximated by enriching the space of the standard finite element functions with additional shape functions that preserve, almost everywhere, the partition of unity property of the finite element shape functions. In the case of a crack, the first term of the crack tip asymptotic field

\footnotetext{
出 $\mathrm{nnn}$

URL: https://sites.google.com/a/unife.it/elena-benvenuti/, http://docente.unife.it/elena.benvenuti (Elena Benvenuti )
}

Preprint submitted to Computer Methods in Applied Mechanics and EngineeringApril 19, 2017 
is usually added to the discretization space 3. However, high-order XFEMs have been developed by enriching the finite element approximation of the nodes surrounding the crack tip with higher-order terms of the crack-tip asymptotic field. For instance, Liu et al. [7 formulated a method with higher-order terms for the direct evaluation of the mixed mode stress intensity factors (SIFs) without extra post-processing. High-order enrichment functions and direct evaluation of Irwin's integral are key in computing mixed-mode stress intensity factors for the XFEM by Lan et al. [8].

Existing XFEM and GFEM applied to fracture mechanics problems exhibit possible loss of accuracy as a consequence of: loss of the partition of unity property in the transition elements, incorrect quadrature, and ill-conditioning 9, 10, 11, 12, 13, 14, 15, 16. For Laborde et al.[10, accuracy is restored through the implementation of high-order polynomial shape functions combined with additional strategies such as the geometrical enrichment of a fixed area containing the crack tip. Examples of blending procedures facing the loss of the partition of unity property in the transition elements are the corrected XFEM [11, and other techniques based on the adoption of ramp-shaped blending functions [12. Another method exempt from the necessity of blending finite elements is the so-called intrinsic XFEM [17] based on the moving least squares functions analogous to those employed in meshless methods. Ill-conditioning can be induced both by the fact that the crack line is close to the finite elements edges, and by the adoption of geometrical-enrichment strategies [10, 15. Stabilized PUFEM methods aim at overcoming ill-conditioning and drawbacks of the blending finite elements by means of suitable assumptions on the space of the enrichments functions. For instance, the stable GFEM (SGFEM) for 2-D and 3-D fracture mechanics problems by Babuška and Banerjee [13, 15, 16] is based on quasiorthogonality between the standard space of the finite element functions and the space of the enrichment functions, and boundedness away from zero of the diagonal elements of the stiffness matrix. Alternative efficient stabilization techniques have also been derived, based either on eigenvalue decomposition of the stiffness matrix [18, or on suitable assumptions of the enrichment functions [19].

It seems that the price to pay to improve accuracy of XFEMs is either a conspicuous increase of the number of unknowns, as in the case of highorder crack tip and/or enrichment functions, or the introduction of special preconditioning procedures of the stiffness matrix. Hence, modified or new stiffness terms appear, whose mechanical meaning seems obscure.

So far, the problem of the convergence of the variational problem adopted in standard XFEM to the singular, asymptotic, solution of the cracked elastic body has been faced from a computational standpoint. On the other hand, the necessity of using suitable variable formulations for cracked bodies has emerged in the context of free-discontinuity problems formulated in the (special) space of bounded variations [20, 21, 22]. In particular, in energy-based formulations for fracture mechanics, the elastic energy of the bulk is separated from that spent on the cracks surface, and Gamma-convergence to Griffith-type fracture energies has been proved [22]. These results obtained for free-discontinuity problems suggest that the classic minimization formulation for non-cracked bodies is no 
longer suited to cracked bodies, and that the singular part of the strain field should be treated separately.

The present paper aims at developing a mechanically consistent XFEM with the same set of enrichments functions as the original XFEM [1, 2, 17, 23. Unlike the original XFEM, the proposed XFEM is based on the mechanical decoupling of the singular and bounded parts of the strain.

To mechanically decouple the singular part of the strain from the other strain terms, the equivalent eigenstrain concept is adopted. In the first decades of the twentieth century [24, 25, 26], Volterra [24, Somigliana [25], and Reißner [26] investigated the equilibrium in the presence of those eigenstrains deriving from the addition or the subtraction of material. The eigenstrain concept was generalized by Colonnetti 27. to any incompatible and non-smooth strain that is not caused by external loads. However, it was Eshelby 28 who formulated a general framework for elastic singularities, stating that inclusions, point and line singularities with infinite self-energies can all be regarded as limiting cases of Somigliana dislocations of finite self-energy. Later, Mura [29, 30, generalized the concept of equivalent eigenstrain for elastic inclusions. Recently, Schmidt et al. 31] have developed an eigendeformation variational formulation that (Gamma-)converges to Griffith's energy.

When the enriched strain field contains Dirac's delta-like terms, previous author's papers [32, 33, 34] have proved that the mechanical decoupling between the singular part of the strain and the other strain terms is necessary to avoid spurious energy contributions. This is the case of strain localization [33, cohesive and finite-thickness interface laws [32, 34. A previous attempt to formulate an equivalent eigenstrain XFEM approach has been done for imperfect interfaces 35 . In the present study, the approach is devised for the case of fracture for the first time.

Generalizing Eshelby's elastic singularities concept 28, the crack is regarded as an elastic singularity in Eshelby's sense by means of the procedures described in Sec. 22 In particular, the displacement field is the sum of a standard term and a term emanating from the crack. The singular strain term is regarded as an eigenstrain, and the associated eigenstress is introduced. Sec. 3 and Sec. 4 illustrate the variational formulation and its discrete version, respectively. It is also shown that, for any non-constant enrichment function, the solving equations of the proposed formulation and that associated with the standard XFEM differ at both the continuum and the discrete level. Sec. 5 assesses the convergence of the computed stress intensity factors to the reference values for a set of plane strain cracked plates. Finally, peculiarities and pros-and-cons of the present formulation are discussed in Sec. 6.

\section{Approximation of the crack as an elastic singularity}

Quoting Eshelby [36], the tip of a crack qualifies as a defect, or an imperfection, in its own right. Therefore, the stress induced by an imperfection can be regarded as some state of internal stress not produced by surface or body forces [37. The aim of this section is to devise an approximated procedure to 
compute the disturbance exerted by the crack within the region $\Omega$ on an applied stress, uniform at large distances. Note that the determination of the analytical solution of Eq. (7) for cracks is out of the aims of the present study. Hence, the path of reasoning of the subsequent developments will deviate from Eshelby's canonical approach [37.

First, in Sec. 2.1 the linear elastic continuum problem with and without cracks is formulated for the plane strain case. The basic statements of Eshelby's approach to the inclusion problem are given in Sec. 2.2 Sec. 2.3 and Sec. 2.4 set the two-fields kinematics of the approach.

\subsection{The linear elastic continuum problem}

Index notation is adopted. A quantity with a repeated superscript is summed over the values $1,2,3$. Differentiation with respect to the coordinate $x_{i}$ is denoted by a subscript, $i$. A small displacement regime is assumed.

The solid $V$ displayed in Fig. 1 is loaded with tractions $p_{i}$ on the region $\partial V_{p}$ of the surface $\partial V$. The body is subjected to vanishing displacements $u_{i}$ on the external surface portion $\partial V_{d}$. In the absence of the crack, for an isotropic material with Young's modulus $E$ and Poisson coefficient $\nu$, the continuum problem is formulated as

$$
\begin{array}{ll}
\sigma_{i j, j}=0, & \text { in } V, \\
\sigma_{i j} n_{j}=p_{i}, & \text { on } \partial V_{p}, \\
u_{i}=0, & \text { on } \partial V_{d}, \\
\sigma_{i j}=\lambda E_{1} \delta_{i j}+2 \mu \frac{1}{2}\left(u_{i, j}+u_{j, i}\right), &
\end{array}
$$

where $\delta_{i j}$ is the Kronecker delta, $E_{1}$ is the first strain invariant and $\lambda=\frac{\nu E}{(1+\nu)(1-2 \nu)}$ and $\mu=\frac{E}{2(1+\nu)}$ are the Lamé constants.

When the body is cracked, stress concentrations and inelastic deformations are expected in the neighborhood of the crack tip. Therefore, the problem of the cracked body has to be cast in the context of the Linear Elastic Fracture Mechanics (LEFM) 38, where the concept of stress intensity factor is key [39, 40. In fact, LEFM computes the asymptotic fields of the stress field in a neighborhood of the crack tip for vanishing distance $r$. Restricting attention to the plane strain crack problem, $u_{1}=u_{1}\left(x_{1}, x_{2}\right), u_{2}=\left(x_{1}, x_{2}\right)$ and $u_{3}$ vanishes. In this case, the equilibrium and constitutive equations reduce to

$$
\begin{aligned}
& \sigma_{\alpha \beta, \alpha}=0, \\
& \frac{1}{2}\left(u_{\alpha, \beta}+u_{\beta, \alpha}\right)=\frac{1+\nu}{E} \sigma_{\alpha \beta}-\frac{\nu}{E} \delta_{\alpha \beta} T_{1},
\end{aligned}
$$

with $\sigma_{33}=\nu \sigma_{\alpha \alpha}$, with $\alpha, \beta=1,2$, and $T_{1}=\sigma_{11}+\sigma_{22}+\sigma_{33}$. The computation of the asymptotic stress and displacement fields is based on the introduction of the Airy stress function. For instance, in a plane strain plate subjected to mode I loading along axis $x_{2}$, the stress field at the crack tip, in a polar system of 
coordinates centered at the crack tip is 41

$$
\begin{aligned}
& \sigma_{22}=\frac{K_{I}}{\sqrt{2 \pi r}} \frac{1}{4}\left(5 \cos \frac{\theta}{2}-\cos \frac{5 \theta}{2}\right), \\
& \sigma_{11}+\sigma_{22}=\frac{K_{I}}{\sqrt{2 \pi r}} 2 \cos \frac{\theta}{2}, \\
& \sigma_{12}=\frac{K_{I}}{\sqrt{2 \pi r}} \frac{1}{4}\left(-\sin \frac{\theta}{2}+\sin \frac{5 \theta}{2}\right),
\end{aligned}
$$

where $K_{I}$ is the mode I stress intensity factor. Stresses (3) are associated with the following displacements 41

$$
\begin{aligned}
& u_{1}=\frac{K_{I}}{\sqrt{2 \pi}} \frac{4\left(1-\nu^{2}\right)}{E} \sqrt{r}\left(1-\frac{\cos ^{2} \frac{\theta}{2}}{2(1-\nu)}\right) \cos \frac{\theta}{2}, \\
& u_{2}=\frac{K_{I}}{\sqrt{2 \pi}} \frac{4\left(1-\nu^{2}\right)}{E} \sqrt{r}\left(1-\frac{\cos ^{2} \frac{\theta}{2}}{2(1-\nu)}\right) \sin \frac{\theta}{2} .
\end{aligned}
$$

For more details on fracture mechanics, reference can be made to Kanninen and Popelar's book 38.

\subsection{Eshelby's eigenstrain approach}

Let an inclusion $\Omega$ of surface $\partial \Omega$ in $V$ be considered. Eshelby's solution of the ellipsoidal inhomogeneity [42] is based on the steps hereinafter described.

I The region $\Omega$ is removed. Thus, it undergoes the strain $-e_{i j}^{*}$. Let $\sigma_{i j}^{*}$ be the stress associated with $-e_{i j}^{*}$ by means of Hooke's law.

II The surface tractions $\sigma_{i j}^{*} n_{j}$ are applied on $\partial \Omega$ to bring $\Omega$ back to its primary shape. The inclusion is rewelded in the matrix. The tractions $\sigma_{i j}^{*} n_{j}$ have originated a layer of body forces $b_{i}^{*}=-\sigma_{i j, j}^{*}$ spread over $\partial \Omega$.

III an opposite tractions field $\sigma_{i j}^{*} n_{j}$ is applied at $\partial \Omega$ so to recover the initial state.

Following Eshelby's seminal work [37] and a later, corrected, version [42], both based on Love's classic treatise 43, the displacement at $\mathbf{r}$ due to a point-force $F_{i}$ at $\mathbf{r}^{\prime}$ writes

$$
U_{i}=U_{i j} F_{j},
$$

where

$$
U_{i j}=\frac{1}{4 \pi \mu} \frac{\delta_{i j}}{\left|\mathbf{r}-\mathbf{r}^{\prime}\right|}-\frac{1}{16 \pi \mu(1-\nu)} \frac{\partial^{2}}{\partial x_{i} \partial x_{j}}\left|\mathbf{r}-\mathbf{r}^{\prime}\right| .
$$

Therefore, the displacement in the inclusion in stage III due to $\sigma_{i j}^{*} n_{j}$ is [37]

$$
u_{i}=\int_{\partial V} \sigma_{j k}^{*} n_{k} U_{i j}\left(\mathbf{r}-\mathbf{r}^{\prime}\right) d S,
$$


where $\sigma_{i j}^{*}$ is the stress associated with $e_{i j}^{*}$ by means of Hooke's law

$$
\sigma_{i j}^{*}=C_{i j h k} e_{h k}^{*} .
$$

Remarkably, Eq. (7) suggests that the compatible strain field $\epsilon_{i j}=\frac{1}{2}\left(u_{i, j}+u_{j, i}\right)$ in the inclusion is, in turn, a function of the eigenstrain $e_{i j}^{*}$.

While the strain in both matrix and inclusion is $\epsilon_{i j}=\frac{1}{2}\left(u_{i, j}+u_{j, i}\right)$, the stress in the matrix writes as

$$
\sigma_{i j}=C_{i j h k} \frac{1}{2}\left(u_{h, k}+u_{k, h}\right) .
$$

The stress in the inclusion is cast as

$$
\sigma_{i j}=C_{i j h k} \frac{1}{2}\left(u_{h, k}+u_{h, k}\right)-\sigma_{i j}^{*},
$$

with $\sigma_{i j}^{*}$ given by Eq. (8).

\subsection{Definition of a two-field-based kinematics}

The crack tip at which the strain and the stress are singular is regarded as a point singularity, and the crack tip is replaced by a region $\Omega$ surrounding the crack tip as shown in Fig. 2

In $\Omega$, the crack is regarded as a disturbance of the strain field. The aim is to correctly capture this disturbance. The first key assumption relies on the approximation of the displacement field as

$$
u_{i}=v_{i}+f a_{i} .
$$

In Eq. (11), $v_{i}$ is the $i$-th component of the displacement field that would be present without the elastic singularity. The term $f a_{i}$ is an additional field incorporating the mathematical structure of the expected solution. Function $f$ features the expected singularity of the displacement field at the crack tip. It is assumed scalar to simplify the subsequent developments. It is also assumed bounded, with singular derivative. Symbol $a_{i}$ denotes the $i$-component of a vector modulating the entity of the disturbance in the space. By construction, $a_{i}$ vanishes where the effects of the elastic singularity vanish while is different from zero where the disturbance is active, i.e. $a_{i}=0$ in $V \backslash \Omega$. Approximation (11) preludes to the XFEM developed in Sect. 4 .

Therefore, the displacement field depends on two distinct vector fields of components $v_{i}$ and $a_{i}$.

By compatibility, for infinitesimal displacements and strains, the total strain is

$$
\frac{1}{2}\left(u_{i, j}+u_{j, i}\right)=\epsilon_{i j}+e_{i j}^{*},
$$

where the strain term

$$
\epsilon_{i j}=\frac{1}{2}\left(v_{i, j}+v_{j, i}+f a_{i, j}+f a_{j, i}\right)
$$


is bounded, while the strain term

$$
e_{i j}^{*}=\frac{1}{2}\left(f_{, i} a_{j}+f_{, j} a_{i}\right)
$$

is singular, because $f$ has singular derivative.

\subsection{Constitutive laws}

The strain field in the whole solid is

$$
\frac{1}{2}\left(u_{i, j}+u_{j, i}\right)=\epsilon_{i j}+e_{i j}^{*},
$$

$\epsilon_{i j}$ and $e_{i j}^{*}$ being defined by Eqs. (13) and (14), respectively.

However, the stress in the inclusion turns out being

$$
\sigma_{i j}=C_{i j h k} \frac{1}{2}\left(u_{h, k}+u_{k, h}\right)-C_{i j h k} e_{h k}^{*}=C_{i j h k} \epsilon_{h k}
$$

in $\Omega$. Furthermore, being $u_{i}$, and thus $\epsilon_{i j}$, function of $e_{i j}^{*}$ through Eqs. (7) and Eq. (8), the stress inside the inclusion is a function of $e_{i j}^{*}$ in its turn, to be cast as

$$
s_{i j}=S_{i j h k} e_{h k}^{*} .
$$

Somehow reminiscent of Eshelby's tensor [37, the constitutive tensor $S_{i j h k}$ will be specified in the forthcoming Sec. 4.2 . Thus, in the inclusion,

$$
\sigma_{i j}=s_{i j}=S_{i j h k} e_{h k}^{*}, \quad \text { in } \Omega .
$$

Equality (18) plays a crucial role in the subsequent developments.

The energy momentum. Eshelby's force on an elastic singularity inside a closed surface $\Gamma$ is the integral

$$
F_{l}=\int_{\Gamma} P_{l j} n_{j} d S,
$$

where $P_{l j}=\mathcal{E} \delta_{l j}-\sigma_{i j} u_{i, l}$ is Eshelby's energy momentum [28, 36], and $\mathcal{E}$ is the strain energy. The classic result [44, 28, 36] that $F_{l}$ vanishes when taken over a closed path $\Gamma$ within which the material is homogeneous and free of defects holds also in the present case. This can be shown by computing

$$
\int_{\Gamma} \mathcal{E} \delta_{l j} n_{j} d S=\int_{\Omega} \mathcal{E}_{, l} d V
$$

where

$$
\mathcal{E}_{, l}=\frac{\partial \mathcal{E}}{\partial \epsilon_{i j}} \epsilon_{i j, l}=\sigma_{i j} u_{i, j l}-\sigma_{i j} e_{i j, l}^{*}=\frac{\partial\left(\sigma_{i j} u_{i, l}\right)}{\partial x_{j}},
$$

because $\sigma_{i j, j}$ by equilibrium, and $e_{i j}^{*}$ vanishes by hypothesis within the considered domain. Hence, the energy momentum $F_{l}$ vanishes, and the exploitation of the J-integral [45] is possible. 


\section{The problem equations}

The forthcoming section 3.1 presents the variational formulation of the proposed approach, while Sec. 3.2 proposes a heuristic approach to the determination of the constitutive tensor $S_{i j h k}$ associated with stress $s_{i j}$ (17). The classic mechanical work used for elastic non-cracked bodies is formulated in Sec. 3.3.

\subsection{Variational formulation of the eigenstrain approach}

The focus of this section is on the problem of the equilibrium of the solid $V$ of Fig. 1 governed by

$$
\begin{array}{ll}
\sigma_{i j, j}=0, & \text { in } V, \\
\sigma_{i j} n_{j}=p_{i}, & \text { on } \partial V_{p}, \\
\sigma_{i j}=s_{i j}, & \text { in } \Omega,
\end{array}
$$

subjected to vanishing displacement on $\partial V_{d}$, and to the constitutive equations (16), 17), 18). A special variational formulation is formulated, whose Euler-Lagrange equations lead to Eqs. (22). To this end, the work-functional

$$
W=\int_{V} \sigma_{i j} \epsilon_{i j} d V+\int_{\Omega} s_{i j} e_{i j}^{*} d V-\int_{\partial V_{p}} p_{i} u_{i} d S
$$

is introduced, where the external work is carried out by tractions $p_{i}$ on the portion $\partial V_{p}$ of the external boundary $\partial V_{p}$. The following problem is formulated. Let $\delta u_{i}$ and $\delta a_{i}$ be admissible variations, being sufficiently differentiable, with vanishing $\delta u_{i}$ on $\partial V_{d}$.

Problem $\mathcal{P}$. Find the stress $\sigma_{i j}$ and $s_{i j}$ such that the first variations of $W$

$$
\left\{\delta W_{v}, \delta W_{a}\right\}
$$

vanish for any virtual admissible variations $\delta u_{i}$ and $\delta a_{i}$, where the compatibility relationships (13) and (14) hold.

Imposition $\delta W=0$ for any admissible variation of the primal fields implies the Euler-Lagrange conditions

$$
\begin{aligned}
& \int_{V} \sigma_{i j, j}\left(\delta v_{i}+f \delta a_{i}\right) d V=0, \\
& \int_{\Omega}\left(\sigma_{i j}-s_{i j}\right)\left(f_{, i} \delta a_{j}+f_{, j} \delta a_{i}\right) d V=0, \\
& \int_{\partial V}\left(\sigma_{i j} n_{j}-p_{i}\right) \delta v_{i} d S=0
\end{aligned}
$$

for any admissible variations $\delta v_{i}$ and $\delta a_{i}$. Because in the present case $f_{, i} \neq 0$, the above stationarity equations 25 imply Eqs. 222. 
3.2. A heuristic approach to the choice of tensor $S_{i j h k}$

From Eqs. (3), it can be drawn that the stress $\sigma_{22}$ across the plane of the crack ahead of the tip and the relative displacement of the crack faces just behind the tip are

$$
\begin{aligned}
\sigma_{22} & =\frac{K_{I}}{\sqrt{2 \pi}} \frac{1}{\sqrt{r}}, \\
\Delta u_{2} & =\frac{8\left(1-\nu^{2}\right)}{E} \frac{K_{I}}{\sqrt{2 \pi}} \sqrt{r} .
\end{aligned}
$$

Fig. 3 reproduces a qualitative picture of the singularity of $\sigma_{22}$ at the crack tip.

To determinate $S_{i j h k}$ (17), a heuristic strategy based on Eqs. (26) is adopted. In particular, it is useful for the subsequent developments to compute the variation of $\Delta u_{2}$ along $x_{1}$

$$
\frac{\partial \Delta u_{2}}{\partial x_{1}}=\frac{4 K_{I}\left(1-\nu^{2}\right)}{E \sqrt{2 \pi x_{1}}} .
$$

Attention is restricted to mode I and to the case where function $f$ in the approximated displacement 11 boils down to

$$
f=\sqrt{r} g_{2}(\theta),
$$

where

$$
g_{2}(\theta)=\left(1-\frac{\cos ^{2} \frac{\theta}{2}}{2(1-\nu)}\right) \sin \frac{\theta}{2} .
$$

At the crack tip, for $\theta=\pi$, the $x_{1}$-displacement component vanishes while the $\mathrm{y}$-component of the opening becomes

$$
u_{2}=v_{2}+\sqrt{r} a_{2} .
$$

At distances $x_{1}$ sufficiently small from the crack tip,

$$
u_{2} \rightarrow \sqrt{x_{1}} a_{2} .
$$

The variation of Eq. (31) is

$$
e^{*}=\frac{a_{2}}{2 \sqrt{x_{1}}} .
$$

For dimensional consistency between Eq. (30) and 4b, $K_{I}$ is assumed to be approximated by $a_{2} E$. Finally, the stress component conjugated with $e^{*} 27$ reads

$$
s=E \frac{\partial \Delta u_{2}}{\partial x_{1}}=\frac{4 K_{I}}{\sqrt{2 \pi x_{1}}}\left(1-\nu^{2}\right)=\frac{4 E}{\sqrt{2 \pi x_{1}}}\left(1-\nu^{2}\right) a_{2}=\frac{8 E}{\sqrt{2 \pi}}\left(1-\nu^{2}\right) e^{*},
$$

where Eq. (32) has been replaced.

According to Eq. (33), the case of single edge notched specimen investigated 


\begin{tabular}{|l|l|l|}
\hline & Standard XFEM & Present XFEM \\
\hline Displacement & $u_{i}=v_{i}+f a_{i}$ & $u_{i}=v_{i}+f a_{i}$ \\
Strain & $\frac{1}{2}\left(u_{i, j}+u_{j, i}\right)=\epsilon_{i j}+e_{i j}^{*}$ & $\frac{1}{2}\left(u_{i, j}+u_{j, i}\right)=\epsilon_{i j}+e_{i j}^{*}$ \\
Stress & $\sigma_{i j}=C_{i j h k}\left(\epsilon_{h k}+e_{h k}^{*}\right)$ & $\sigma_{i j}=C_{i j h k} \epsilon_{h k}$ \\
Internal work & $\int_{V} \sigma_{i j}\left(\epsilon_{i j}+e_{i j}^{*}\right) d V$ & $\int_{V} \sigma_{i j} \epsilon_{i j} d V+\int_{V} s_{i j} e_{i j}^{*} d V$ \\
\hline
\end{tabular}

Table 1: Comparison between the standard formulation and the present formulation

in Sec. 5.2 is solved by assuming the following expression of $S_{i j h k}$

$$
S_{i j h k}=\frac{8\left(1-\nu^{2}\right)}{\sqrt{2 \pi}} C_{i j h k} .
$$

For the case of the central crack studied in Sec. $5.4, S_{i j h k}$ has been approximated as

$$
S_{i j h k}=\frac{8\left(1-\nu^{2}\right)}{\sqrt{2 \pi}}\left(\cos ^{2}(\alpha)+\gamma \sin ^{2}(\alpha)\right) C_{i j h k},
$$

$\alpha$ being the inclination of the crack with respect to the loading direction.

In the applications described in Sec. 5 parameter $\gamma$ is set equal to 0 when pure mode I opening is expected, while it is non-vanishing for mixed shearingopening mode.

\subsection{Variational formulation based on the classic definition of the mechanical} work for elastic bodies

Following the classic approach adopted for linear elastic bodies, the total work writes

$$
W=\int_{V} \sigma_{i j}^{\prime}\left(\epsilon_{i j}+e_{i j}^{*}\right) d V-\int_{\partial V_{p}} p_{i} u_{i} d S,
$$

where the stress is $\sigma_{i j}^{\prime}=C_{i j h k}\left(\epsilon_{h k}+e_{h k}^{*}\right)$. Imposition of the stationarity equations for any virtual variation $\delta u_{i}$ and $\delta a_{i}$ leads to the system of equations

$$
\begin{array}{ll}
\sigma_{i j, j}^{\prime}=0 & \text { in } V, \\
f \sigma_{i j, j}^{\prime}=0 & \text { in } V, \\
\sigma_{i j}^{\prime} n_{j}=p_{i} & \text { on } \partial V_{p} .
\end{array}
$$

It can be observed that the mechanical meaning of Eq. (37b) is not evident. Obviously, Eqs. (37a)-(37b) become linearly dependent if $f$ is a constant, not a piecewise constant, function. However, in this case, the classic Finite Element method should be used, and the adoption of the special kinematics (11) is not justified.

Tab 1 contains a prospective view of the main equations of the proposed approach versus the standard one. 


\section{Approximation of the problem}

The present section contains the basic relationships useful to transform in their discrete counterpart stresses and strains (Sec. 4.1)), and the variational formulation (Sec. 4.2). The standard XFEM discrete variational formulation is obtained in Sec. 4.3 . Sec. 4.4 puts into evidence the effects of the assumed variational formulation on accuracy and blending.

A bold notation denotes vectors and matrices.

\subsection{Discrete strain and stress fields}

The first term of the displacement (11) is approximated by interpolating the nodal vector $\mathbf{v}_{i}$ of the generic $i$-th node, with $i=1, \ldots, N$, through $N$ bilinear finite element shape functions $\mathcal{N}_{i}$

$$
\mathbf{v} \approx \sum_{i=1}^{N} \mathcal{N}_{i}(\mathbf{x}) \mathbf{v}_{i}
$$

The vector field $f \mathbf{a}$ is approximated by means of

$$
f \mathbf{a} \approx \sum_{i=1}^{N} \sum_{k=1}^{N_{k}} \mathcal{N}_{i}(\mathbf{x}) \mathfrak{f}_{k}(r(\mathbf{x}), \theta) \mathbf{a}_{i k}
$$

where $N$ is the number of the finite element nodes, and $N_{k}$ is the number of enrichment functions. Among the possible choices for the crack tip functions [16, in the present paper, the first-order crack tip functions [1]

$$
\left(\mathfrak{f}_{1}, \mathfrak{f}_{2}, \mathfrak{f}_{3}, \mathfrak{f}_{4}\right)=\left(\sqrt{r} \sin \frac{\theta}{2}, \sqrt{r} \cos \frac{\theta}{2}, \sqrt{r} \sin \frac{\theta}{2} \sin \theta, \sqrt{r} \cos \frac{\theta}{2} \cos \theta\right)
$$

are assumed. The approximating space contains also discontinuous functions, such as the Heaviside function $\mathcal{H}$, reflecting the displacement discontinuity along the crack line. Finally, the displacement field is approximated by means of the following expression 23 .

$$
\mathbf{u}(\mathbf{x})=\sum_{i=1}^{N} \mathcal{N}_{i}(\mathbf{x}) \mathbf{v}_{i}+\sum_{I=1}^{N} \mathcal{N}_{i}(\mathbf{x}) \mathcal{H}(\mathbf{x}) \mathbf{j}_{i}+\sum_{i=1}^{N} \sum_{k=1}^{N_{k}} \mathcal{N}_{i}(\mathbf{x}) \mathfrak{f}_{k}(r(\mathbf{x}), \theta) \mathbf{a}_{i k},
$$

Typically, the approximated domain is split into three sets, namely the set of the finite elements whose nodes are not enriched, the set of the partially enriched elements, called transition elements, and the set of the elements whose nodes are totally enriched. Consequently, the line across which the displacement is discontinuous is replaced by a finite element layer, that, in Fig. 4 is delimited by the circled nodes that are enriched with the Heaviside function. The presence of the crack tip is instead taken into account through a crack-tip-enriched finite element, whose nodes are marked with a square in Fig. 4. 
with

$$
\begin{aligned}
& \boldsymbol{\epsilon}=\mathbf{B} \mathbf{v}+\tilde{\mathbf{F}} \mathbf{B}_{M} \mathbf{a}, \\
& \mathbf{e}^{*}=\mathbf{B}_{\mathbf{F}} \mathbf{a} .
\end{aligned}
$$

Matrices $\mathbf{B}_{M}$ and $\mathbf{B}_{\mathbf{F}}$ are associated with the gradient of $\mathbf{M}$ and with the tensor product $\nabla \mathbf{F} \otimes \mathbf{N a}$, respectively. Moreover, $\tilde{\mathbf{F}}$ contains the crack tip enrichment functions but does not coincide with $\mathbf{F}$ for dimensional consistency reasons. More details on the structure of the aforementioned matrices are in the appendix. Finally, the discrete form of the stresses is

$$
\begin{aligned}
& \boldsymbol{\sigma}=\mathbf{C B v}+\mathbf{C} \tilde{\mathbf{F}} \mathbf{B}_{M} \mathbf{a}, \\
& \mathbf{s}=\mathbf{S B}_{\mathbf{F}} \mathbf{a}
\end{aligned}
$$

are introduced, where $\mathbf{S}$ is given by Eq. 35 .

\subsection{The discrete variational formulation of the eigenstrain approach}

After replacement of Eqs. (45), the discrete form of the proposed total virtual work reads

$$
W=\int_{V} \boldsymbol{\sigma} \cdot\left(\mathbf{B} \delta \mathbf{v}+\tilde{\mathbf{F}} \mathbf{B}_{M} \delta \mathbf{a}\right) d V+\int_{V} \mathbf{s} \cdot \mathbf{B}_{\mathbf{F}} \delta \mathbf{a} d V-\int_{\partial V_{p}} \mathbf{p} \cdot \mathbf{N} \delta \mathbf{v} d V
$$

for any virtual $\delta \mathbf{v}$ and $\delta \mathbf{a}$ that vanish at the boundaries, being $\boldsymbol{\sigma}$ and s given by Eqs. (46). The solving equations are obtained by computing the discrete form 
of the Euler-Lagrange conditions for $W$

$$
\begin{aligned}
& \int_{V} \mathbf{B}^{T} \boldsymbol{\sigma} d V=\int_{V} \mathbf{N}^{T} \mathbf{p} d S, \\
& \int_{V}\left(\tilde{\mathbf{F}} \mathbf{B}_{M}\right)^{T} \boldsymbol{\sigma} d V+\int_{V} \mathbf{B}_{\mathbf{F}}^{T} \mathbf{s} d V=\mathbf{0} .
\end{aligned}
$$

Therefore, the stiffness matrix is

$$
\mathbf{K}=\left(\begin{array}{ll}
\int_{V} \mathbf{B}^{T} \mathbf{C B} d V & \int_{V} \mathbf{B}^{T} \mathbf{C} \tilde{\mathbf{F}} \mathbf{B}_{M} d V \\
\int_{V}\left(\tilde{\mathbf{F}} \mathbf{B}_{M}\right)^{T} \mathbf{C B} d V & \int_{V}\left(\tilde{\mathbf{F}} \mathbf{B}_{M}\right)^{T} \mathbf{C} \tilde{\mathbf{F}} \mathbf{B}_{M} d V+ \\
& \int_{V} \mathbf{B}_{\mathbf{F}}^{T} \mathbf{S B}_{\mathbf{F}} d V
\end{array}\right)
$$

where $\mathbf{S}$ follows from Eq. (34).

\subsection{The standard discrete formulation of XFEM}

Let the approximating space be the same as that introduced in the previous section. For the standard XFEM approach, the discrete form of the virtual work function is

$$
W^{\prime}=\int_{V} \boldsymbol{\sigma}^{\prime} \cdot\left(\mathbf{B} \delta \mathbf{v}+\tilde{\mathbf{F}} \mathbf{B}_{M} \delta \mathbf{a}+\mathbf{B}_{\mathbf{F}} \delta \mathbf{a}\right) d V-\int_{\partial V_{p}} \mathbf{p} \cdot \mathbf{N} \delta \mathbf{v} d V
$$

for any virtual $\delta \mathbf{v}$ and $\delta \mathbf{a}$, where $\boldsymbol{\sigma}^{\prime}=\mathbf{C}\left(\mathbf{B} \delta \mathbf{v}+\tilde{\mathbf{F}} \mathbf{B}_{M} \delta \mathbf{a}+\mathbf{B}_{\mathbf{F}} \delta \mathbf{a}\right)$.

The stationarity equations of $W^{\prime}(50)$ are

$$
\begin{aligned}
& \int_{V} \mathbf{B}^{T} \boldsymbol{\sigma}^{\prime} d V=\int_{\partial V_{p}} \mathbf{N}^{T} \mathbf{p}, \\
& \int_{V}\left(\tilde{\mathbf{F}} \mathbf{B}_{M}\right)^{T} \boldsymbol{\sigma}^{\prime} d V+\int_{V} \mathbf{B}_{\mathbf{F}}^{T} \boldsymbol{\sigma}^{\prime} d V=\mathbf{0} .
\end{aligned}
$$

The associated stiffness matrix is

$\mathbf{K}^{\prime}=\left(\begin{array}{ll}\int_{V} \mathbf{B}^{T} \mathbf{C B} d V & \int_{V}\left(\mathbf{B}^{T} \mathbf{C} \tilde{\mathbf{F}} \mathbf{B}_{M}+\mathbf{B}^{T} \mathbf{C B}_{\mathbf{F}}\right) d V \\ \int_{V}\left(\left(\tilde{\mathbf{F}} \mathbf{B}_{M}\right)^{T} \mathbf{C B} d V+\mathbf{B}_{\mathbf{F}}^{T} \mathbf{C B}\right) d V & \int_{V}\left(\left(\tilde{\mathbf{F}} \mathbf{B}_{M}\right)^{T} \mathbf{C} \tilde{\mathbf{F}} \mathbf{B}_{M}+\mathbf{B}_{\mathbf{F}}^{T} \mathbf{C B}_{\mathbf{F}}\right) d V+ \\ & \int_{V}\left(\mathbf{B}_{\mathbf{F}}^{T} \mathbf{C} \tilde{\mathbf{F}} \mathbf{B}_{M}+\left(\tilde{\mathbf{F}} \mathbf{B}_{M}\right)^{T} \mathbf{C B}_{\mathbf{F}}\right) d V\end{array}\right)$.

The terms of the stiffins

The terms of the stiffness matrix (52) containing the singular part of the strain are coupled with those containing the bounded part of the strain irrespectively of the mechanical meaning associated with each of these strain contributions. A comparison between Eq. (48b) and Eq. (51b) suggests that the present formulation coincides with the standard XFEM for any vanishing $\mathbf{B}_{F}$.

Let Eq. (51b) be rewritten as

$$
\int_{V}\left(\left(\tilde{\mathbf{F}} \mathbf{B}_{M}\right)^{T}+\mathbf{B}_{\mathbf{F}}^{T}\right) \boldsymbol{\sigma}^{\prime} d V=\mathbf{0} .
$$




\begin{tabular}{|c|c|c|c|}
\hline Type & $f$ & $\mathbf{B}_{M}$ & $\mathbf{B}_{F}$ \\
\hline $\begin{array}{l}\text { Discontinuity } \\
\text { Regularized discontinuity } \\
\text { weak discontinuity } \\
\text { crack tip }\end{array}$ & $\begin{array}{l}\mathcal{H} \\
\mathcal{H}_{\rho} \\
\operatorname{sign}(x) \\
\sqrt{x}\end{array}$ & $\begin{array}{l}\mathcal{H}\left[-\frac{1}{h}, \frac{1}{h}\right] \\
\mathcal{H}_{\rho}\left[-\frac{1}{h}, \frac{1}{h}\right] \\
\operatorname{sign}(x)[-1 / h 1 / h] \\
\sqrt{x}\left[-\frac{1}{h}, \frac{1}{h}\right]\end{array}$ & $\begin{array}{l}- \\
\delta_{\rho}\left[-\frac{1}{h}, \frac{1}{h}\right] \\
\pm 1\left[1-\frac{\bar{x}}{h}, \frac{\bar{x}}{h}\right] \\
\frac{1}{2 \sqrt{x}}\left[1-\frac{\bar{x}}{h}, \frac{\bar{x}}{h}\right]\end{array}$ \\
\hline
\end{tabular}

Table 2: Representative one-dimensional cases of enrichments for $P_{1}$ finite element approximation functions

The latter equation is quite restrictive to be satisfied as it has to be satisfied not only within the enriched finite element at the crack tip but also in the adjacent finite elements, the so called transition elements, where the partition of unity property is lost. From a general standpoint, the structure of Eq. 53 holds not only for the crack tip enrichment but also for any set of enrichment functions.

\subsection{Remarks on the effects of the assumed variational formulation on accuracy and blending}

We resume the path of reasoning started in references [9, 10, 12, 46 regarding the loss of accuracy, and related possible remedies, associated with transition elements. A generic one-dimensional transition finite element of length $h$ is considered in a uniform mesh, being $x$ is the distance from the assumed singularity $f$, such as a discontinuity of displacements, a material change, or a crack tip. For a linear, $P_{1}$, finite element approximation, the local shape functions are $N_{1}=1-\bar{x} / h$ and $N_{2}=\bar{x} / h$, where $\bar{x}$ is the local abscissa from node 1 with $x_{1}$ to node 2 with $x_{2}$. In the considered transition element, the lack of partition of unity has consequences not only on the approximation of the displacement field but also on the approximation of the strain field. The reason is that the strain $\epsilon_{11}$ writes

$$
\epsilon_{x x}=\frac{1}{h}\left(v_{2}-v_{1}\right)-\frac{a_{1}}{h} f(x)+a_{1}\left(1-\frac{\bar{x}}{h}\right) f^{\prime}(x) .
$$

The "intruder" in Eq (54) is the term $\frac{a_{1}}{h} f(x)$. It is an unwanted term, because it represents a strain component that does not match with the searched strain field, whose profile should rather be ruled by the term with $f^{\prime}(x)$. It seems, therefore, reasonable that the practice of computing the quadratic form of the strain energy will introduce a series of terms where the unwanted term is coupled with the others, with a consequent possible loss of accuracy. This fact can be read in two distinct ways: as a numerical evidence to be faced by means of high order enrichment of shape functions 9 , 10, 46, 12, 47, and as a lack of mechanical consistency [32, 48, 33, 34, 35]. To this author's knowledge, the present study is the first to adopt the latter standpoint in fracture mechanics.

The main numerical consequence of the adopted approach is the elimination of the coupling of the term with $f^{\prime}$ with the term with $f$ and the standard displacement term, in the one-dimensional case, namely between matrix $\mathbf{B}_{F}$ and matrices $\mathbf{B}$ and $\tilde{\mathbf{F}} \mathbf{B}_{M}$ in the general case where $\mathbf{F}$ is a vector of enrichment functions. However, let us keep the one-dimensional format to simplify the 
reasoning. One of the crucial relationships of the present XFEM is Eq. (22c), whose discrete one-dimensional expression reduces to

$$
E \frac{v_{2}-v_{1}}{h}-E \frac{a_{1}}{h} f(x)=\bar{E} a_{1}\left(1-\frac{\bar{x}}{h}\right) f^{\prime}(x),
$$

where $E$ is the Young's modulus and $\bar{E}$ is a function of $E$ depending on the type of problem to be studied. In fact, the present XFEM does not impose Eq. 22c in a strong form but in the weak form of Eq. (48b). However, the strong form (55) suggests some remarks.

In particular, Eq. (55) specializes as follows

- for the displacement discontinuity Heaviside enrichment $\mathcal{H}$,

$$
\frac{v_{2}-v_{1}}{h}-\frac{a_{1}}{h} \mathcal{H}(x)=0 ;
$$

- for the regularized displacement discontinuity enrichment $\mathcal{H}_{\rho}[32$,

$$
\frac{v_{2}-v_{1}}{h}-\frac{a_{1}}{h} \mathcal{H}_{\rho}(x)=\frac{1}{t} a_{1}\left(1-\frac{\bar{x}}{h}\right),
$$

where $t$ is a unit length to be introduced for dimensional consistency;

- for the material discontinuity enrichment $\operatorname{sign}(x)$,

$$
\frac{v_{2}-v_{1}}{h}-\frac{a_{1}}{h} \operatorname{sign}(x)= \pm a_{1}\left(1-\frac{\bar{x}}{h}\right) ;
$$

- for the crack tip enrichment $\sqrt{x}$

$$
\frac{v_{2}-v_{1}}{h}-\frac{a_{1}}{h} \sqrt{x}=a_{1} \frac{8}{\sqrt{2 \pi}}\left(1-\frac{\bar{x}}{h}\right) \frac{1}{2 \sqrt{x}} .
$$

Eq. (59) contains the mesh size of the uniform mesh. Under the assumption that $x_{1}=h$, and that the transition element is placed immediately at the right of the reproducing finite element enriched with the crack tip enrichment functions, the trends of the term $\sqrt{x}$, that is independent of the discretization, and the term $\left(1-\frac{\bar{x}}{h}\right) \frac{1}{2 \sqrt{x}}$, that depends on $h$, are compared in Fig. 5 . The bigger the mesh size the better the strong form (59) is satisfied in the transition element, because, there, the terms have comparable trends. For small mesh sizes, the differences between the profiles in the transition element are enhanced. Hence, a fast convergence for coarse meshes is expected.

Furthermore, it can be observed in Tab. 2 that coupling $\mathbf{B}_{F}$ with $\mathbf{B}_{M}$ leads to terms containing $\bar{x} / h$, that do not vanish for decreasing $h$. On the other hand, coupling $\mathbf{B}_{F}$ with $\mathbf{B}$ leads to terms of the same order as $\mathbf{B}_{F}$. As shown in Fig. (5), the terms of $\mathbf{B}_{F}$ evaluated in the first element close to the crack tip decrease for decreasing mesh size. Hence, the contribution to the global stiffness matrix deriving from coupling $\mathbf{B}_{F}$ and $\mathbf{B}$ is expected to decrease for 
decreasing $h$. This implies that the discrepancy between the solution obtained by means of the proposed XFEM and that obtained by means of the standard XFEM should decrease for increasing mesh size. Nevertheless, the two solutions will be different, because matrix $\mathbf{S}$ of the eigenstrain approach appears only in the proposed XFEM.

The case of the material discontinuity. As a collateral remark, one can observe that, for the present 1D element placed at the right of the singularity distant $x_{1}$ from node 1, Eq. (58) writes

$$
\frac{v_{2}-v_{1}}{h}-\frac{a_{1} x}{h}-a_{1} \frac{1}{h}+\frac{a_{1} x}{h}-\frac{a_{1} x_{1}}{h}=\frac{v_{2}-v_{1}}{h}-a_{1} \frac{1}{h}-\frac{a_{1} x_{1}}{h} .
$$

Unlike in standard XFEM, in the present case, the vanishing of the linear term makes convergence to a homogeneous strain possible. The implementation of this case of material discontinuity is left for a forthcoming application.

\section{Applications}

After Sec5.1 introducing the main general features of the adopted implementation strategy, Sec. 5.2, 5.3 , and 5.4 show the results obtained in the simulation of a single edge notched test and a tensile specimen with an inclined central crack.

\subsection{Premises}

As previously mentioned, this study intentionally pursues the simplest possible procedures of implementation. Thus, bilinear shape functions $\mathcal{N}$ have been used, and Gauss integration at the crack tip element has been kept. However, it is known 10, 11, 49 that the adoption of polar quadrature rules can overcome the lack of accuracy in the integration of the stiffness contributions of the crack tip enriched finite element.

The stress intensity factors have been obtained from the computation of the J-integral using domain forms of the interaction integrals [1]. The J-integral domain radius associated with an enriched element of area $\mathrm{A}$ is $3 \sqrt{A}$. Other techniques are described in Sukumar's et al. review [49. For example, Song et al. 50. based the determination of the stress intensity factors on the computation of Irwin's integral [40].

In the next developments, the so-called topological instead of the so-called geometrical enrichment [10, 16] is adopted. It has been ascertained [10, 16] that topological enrichment decreases accuracy while keeping the stiffness matrix well-conditioned. Alternatively, a certain number of finite elements within a fixed area in front of the crack tip is enriched. This is called geometrical enrichment. Besides increasing the accuracy, geometrical enrichment increases the conditioning number of the stiffness matrix. Hence the necessity of stabilization of the formulation follows, such as those described in [13, 15]. 
The conditioning number of the stiffness matrix obtained with the present formulation is the same as that obtained in first-order standard XFEM with topological enrichment. The condition number have not been reported as they appear not a major issue in topological enrichments, contrarily to geometrical enrichment.

Uniform meshes made of four-nodes elements have been adopted. Meshes have not been refined around the crack.

The forthcoming sections contain a critical comparison among the results obtained by means of the proposed formulation, those obtained by employing the standard XFEM approach, and the reference results. Accuracy is assessed for variable crack length and inclination angles.

\subsection{Single edge notched specimen}

The first example is the Single Edge Notched (SEN) plate in plane strain state subjected to a uniform tensile stress $p=1 \mathrm{MPa}$ illustrated in Fig. 66. The plate has width $W=3 \mathrm{~cm}$ and height $H=6 \mathrm{~cm}$. A variable crack length $a$ has been considered. The stress intensity factor of the single-edge notched specimen has been computed and compared with Tada's formula [51]

$$
K_{I}^{r e f}=p \sqrt{\pi a} \sqrt{\frac{2 W}{\pi a} \tan \frac{\pi a}{2 W}} \frac{0.752+2.02 \frac{a}{W}+0.37\left(1-\sin \frac{\pi a}{2 W}\right)}{\cos \frac{\pi a}{2 W}} .
$$

Among all, Tada's formula is the most accurate, with an accuracy better than $5 \times 10^{-3}$ for any $a / W$. The matrix of the cartesian components of tensor $S_{i j h k}$ has been assumed according to Eq. (34).

First, the case in which the crack tip falls at the center of the finite element is studied. In Figs. $7 \mathrm{a}, 8 \mathrm{a}$, and $9 \mathrm{a}$, the relative error in normalized discrete $\mathbb{L}^{2}(V)$ norm of $K_{I}$ is shown. Figs. 7b, 8p, and $9 \mathrm{p}$ display the values of the ratio $K_{I} / K_{I}^{r e f}$ for variable mesh size, showing that convergence is from below. The crack lengths are $a=2 W / 3, a=W / 2$, and $a=W / 6$, respectively. Red squares and green circles denote the results obtained by means of the standard XFEM, and those obtained by means of the present XFEM, respectively. The present XFEM is one order of magnitude more accurate than the standard XFEM. The slopes of the $\mathbb{L}^{2}(V)$-error profiles have been pointed out in the logarithmic scale.

The relative error of the case where the crack is aligned along the element edges at distance $H / 2$ from the bottom is shown in Fig. 10 for $a=0.5 W$ (a) and $a=0.6 \mathrm{~W}$. In this special case, two transition elements are semi-enriched at the crack tip. This seems to have the same effect on accuracy as that produced by a geometrical enrichment. Here, the gain in accuracy of the present XFEM is evident for decreasing mesh size.

Then, the case where the crack tip position is randomly placed within the finite element is addressed. Fig. 11 illustrates the case of $a=0.8 \mathrm{~W}$. Smaller crack lengths such as $a=0.45 \mathrm{~W}$, and $a=0.175 \mathrm{~W}$ displayed in Figs. 12 are associated with errors with an oscillatory trend due to the fact that, when the crack tip approaches the element edges, accuracy deteriorates. Nevertheless, the 
errors of the proposed XFEM are generally smaller than that of the standard XFEM. In particular, the sensitivity of the proposed method to the crack tip position is illustrated in Fig. 13 considering $a=0.45 \mathrm{~W}$ and three meshes with $h=1 / 9,1 / 19,1 / 29 \mathrm{~cm}$. The crack tip positions change depending on the mesh size as shown in Figs. 13 -c. In these figures, the J-integral-domain is shown in red. Figs. $13 \mathrm{~d}-\mathrm{f}$, and $13 \mathrm{~s}$-h refer to the proposed XFEM and to the standard XFEM, respectively.

To investigate the local behavior of the computed stress profiles, the stress components have been compared with that obtained by means of the standard XFEM. Figs. 14 display the $\sigma_{22}, \sigma_{11}$, and $\sigma_{12}$ components, where the $y$-axis is parallel to the loading direction and the $x$ axis is orthogonal. A mesh of $57 \times 114$ finite elements has been used. The contour plots are similar, but the present XFEM typically reproduces the eigenstrain shape within the finite element at the crack tip.

\subsection{Tensile plate with central horizontal crack}

A CCT test of a plate in plane strain state subjected to a tensile loading of $p=1 \mathrm{MPa}$ with a central horizontal crack is considered. The plate is a square of edge length $W=10 \mathrm{~cm}$. The geometry can be inferred from Fig. 15 for $\alpha=0^{\circ}$. The plate is loaded by a tensile distributed load of $p=1 \mathrm{MPa}$, and is made of isotropic material with Young modulus $E=100 \mathrm{MPa}$ and Poisson coefficient $\nu=0.3$. Based on geometry and loading condition, the stress intensity factor of reference is 51 .

$$
K_{I}^{r e f}=p \sqrt{\pi a}\left(1-0.025\left(\frac{a}{W}\right)^{2}+0.06\left(\frac{a}{W}\right)^{4}\right) \sqrt{\sec \frac{\pi a}{2 W}} .
$$

Matrix $\mathbf{S}$ is given by Eq. (34).

Fig. 16 displays the $\mathbb{L}^{2}(V)$ norm of the error on the computation of the relative error in normalized discrete $\mathbb{L}^{2}(V)$ norm of $K_{I}$ (a) and ratio $K_{I} / K_{I}^{\text {ref }}$ (b) for variable mesh size and $2 a=8 W / 100$. Green squares and red circles denote the proposed XFEM and the standard XFEM, respectively.

\subsection{Slanted central crack}

The third example is the square plate of Sec. 5.3 and displayed in Fig. 15 but with the central crack of length $2 a$ inclined of $\alpha$. For an infinite plate subjected to a plane strain state, the analytical solution predicts, for an infinite plate, the following values of the stress intensity factors

$$
K_{I}^{r e f}=p \sqrt{\pi a} \cos ^{2} \alpha, \quad K_{I I}^{r e f}=p \sqrt{\pi a} \sin \alpha \cos \alpha .
$$

In the present CCT test, the matrix of the cartesian components of tensor $S_{i j h k}$ (35) has been assumed according to Eq. (35) with parameter $\gamma$ set equal to 100 . This $\gamma$ value leads to the best approximation of both $K_{I}$ and $K_{I I}$. In fact, greater values do not improve significantly the approximation of $K_{I I}$, while 
deteriorate the accuracy of the approximation of $K_{I}$; smaller values increase the accuracy of $K_{I}$ while lead to unsatisfying values of $K_{I I}$.

Figs. 17 and 18 show the values of $K_{I}$ and $K_{I I}$ computed for variable inclination $\alpha$ and crack lengths $2 a=W / 10$ and $2 a=W / 25$ by means of the proposed XFEM (green circles and triangles), and through the standard XFEM (red squares and diamonds). The continuous black lines denote the reference values (63), where for $\alpha=0$ the reference formula $(62)$ has been exploited, being more accurate than Eq. 63). In particular, circles and squares denote $K_{I^{-}}$-values computed with the present XFEM and the standard XFEM, respectively, while triangles and diamonds indicate $K_{I I^{-}}$values evaluated through the present and the standard XFEM, respectively.

Fig. 19 illustrates the relative error in normalized discrete $\mathbb{L}^{2}(V)$ norm of $K_{I}$ (a), $K_{I I}$ (c), $K_{I} / K_{I}^{r e f}(\mathrm{~b})$, and $K_{I I} / K_{I I}^{r e f}$ (d) for variable mesh size with fixed crack length $2 a=3 W / 100$ and inclination $\alpha=\pi / 4$. Green squares and red circles denote the proposed XFEM and the standard XFEM, respectively. Note that convergence is from above. Figs. 20 and 21 display the contour plots of $\sigma_{22}, \sigma_{11}, \sigma_{12}$ using the standard XFEM (a) and the present XFEM (b). In particular, in Fig. 20, $2 a=W / 10, \alpha=40^{\circ}$ and a mesh of $49 \times 49$ elements have been adopted, while Fig. 21 has been obtained for $2 a=W / 50, \alpha=80^{\circ}$, and a mesh of $199 \times 199$ elements. It can be noted that the disturbance of the stress field around the crack is more localized in the present XFEM than in the standard one.

\section{Discussion}

After a critical discussion of the peculiarities of proposed XFEM with respect to the standard XFEM in Sec. 6.1. Secs 6.2 and 6.3 highlight the main pros and cons of the proposed approach, based on the results of Sec. 5 .

\subsection{Differences with respect to the standard XFEM}

The present and the standard XFEMs are based on different solving equations at both the continuum and the discrete level. In particular, in Eq. 49), the asymptotic enrichment functions are not coupled with the other terms. Moreover, the term of the asymptotic enrichment function in Eq. 44 contains the constitutive matrix $\mathbf{S}$, while in the homologous term of Eq. (52) the elastic constitutive matrix $\mathbf{E}$ appears.

In particular, the stiffness matrix of the present method can indeed be obtained from the standard one after replacement of the constitutive tensor $\mathbf{S}$ where required and removal of certain coupling stiffness terms, as evident from a comparison between Eq. (49) and Eq. (52).

However, when the derivative of the enrichment function $f$ vanishes, such as in the case of the Heaviside function, the two stiffness matrices coincide, though, in this case, the equivalent eigenstrain approach ceases to be meaningful.

Therefore, the proposed and the standard eXtended Finite Element methods share the same approximating space, and differ in the variational approach and 
the solving equations. Only for a piecewise constant function $f$, the proposed formulation reduces to the standard XFEM. Nevertheless, for decreasing mesh size, some terms of the stiffness matrix of the standard XFEM tend to decrease, and the difference between the stiffness matrices diminishes for decreasing mesh size.

\subsection{Advantages}

For the SEN test of Sec. 5.2, the stress intensity factors computed through the present XFEM are one order of magnitude more accurate than those obtained with first-order non-stabilized XFEM of the mainstream school [1. The gain in accuracy is evident especially when the mesh is coarse, while it attenuates for decreasing mesh size. In particular, crack tips falling within the finite element lead to the highest accuracy.

In the CCT test of Secs. 5.3 and 5.4 the main result is the robustness of the present XFEM for variable crack position and length. Figs. 17 19] show that the accuracy of the proposed approach in evaluating $K_{I I}$ is higher than that of the standard XFEM when short crack lengths are considered. As for the accuracy of evaluation of $K_{I}$, the present approach is more robust when meshes are coarse and the crack lengths are short compared to the plate edge. Figs. 17 and 18 show that there are indeed positions of the crack with respect to the plate for which the standard XFEM diverges, while the present XFEM still converges to satisfying values. A further advantage is the fact that the additional solving equation associated with the enrichment field has a clear mechanical meaning.

It is known from previous studies that convergence can be improved by adopting high-order polynomial shape functions and low-order crack tip functions [52, 10, 46] or high-order crack tip enrichment functions [8]. For the slanted crack case, the proposed low-order XFEM exhibits results analogous to that obtained by Lan et al. 8 by means of crack tip functions of the second order. For the single edge crack case, the effect on the accuracy of the present low-order XFEM is analogous to that obtained by XFEMs with high-order polynomial shape functions [10, 46, 47, however, without increasing the condition number of the solving system.

\subsection{Limitations}

Being simplified, the proposed implementation strategy has certain limitations. Based on the fact that the standard Heaviside function is used, the proposed procedure is affected by the same sensitivity to the position of the discontinuity line as non-stabilized XFEM. When the crack tip is randomly placed with respect to the finite element, convergence of the stress intensity factors is oscillatory, though the proposed XFEM remains more accurate. Laborde et al. 10] detected similar oscillations in their high-order geometrically enriched XFEM, and explained them as oscillations around the exact values. The regularized Heaviside function together with the equivalent eigenstrain procedure described in [35] can be used instead of the standard Heaviside to get wellconditioned matrices when the crack line is close to the edges of the element. 
It is indeed remarkable that the regularized XFEM developed in [35] does not need a special treatment of blending elements. Moreover, the choice of the tensor $S_{i j h k}$ has been done on a heuristic basis. Although the present results show that such a simplified choice leads to quite effective results, it can be argued that a more rigorous derivation could further improve the accuracy. Finally, high order enrichments and geometrical crack-tip enrichment [10, 47, 15] have not been used. Obviously, the implementation of enhanced procedures, such as stabilization or geometrical enrichment, is possible also in the present XFEM.

As for the convergence rate, the present formulation, unfortunately, does not always increase the slope of the $K_{I}$ and $K_{I I}$ error profiles significantly. It should be however kept in mind that the reference values are not exact values, with an approximation of the $5 \%$. The analytical boundary conditions [10 could rather be used.

\section{Conclusions}

The present study proposes a novel XFEM for the determination of stress intensity factors with a minimal effort of implementation. The XFEM has been considered not only in relation with a functional space but also with a variational principle, where the singular part of the strain is regarded as an equivalent eigenstrain. For any non-constant enrichment function, the solving equations of the proposed formulation and that associated with the standard XFEM differ at both the continuum and the discrete level. For the simulated plane strain examples, the present results show that stress intensity factors are almost one order of magnitude more accurate than those obtainable through first-order non-stabilized XFEM with topological enrichment. Furthermore, the present XFEM exhibits excellent robustness for variable crack geometry. The gain in accuracy and robustness is more evident for coarse meshes. Based on this study, the proposed XFEM is competitive with enhanced XFEMs, while keeping a minimal computational burden.

Acknowledgments. The Matlab (C)XFEM code of Mattew Pais freely available at http://www.matthewpais.com has been modified by the author to obtain the present XFEM.

\section{Appendix A.}

In the two-dimensional case, vector $\mathbf{a}$ is

$$
\mathbf{a}^{T}=\left[a_{11}^{x}, a_{11}^{y}, a_{12}^{x}, a_{12}^{y}, \ldots, a_{1 N_{k}}^{x}, a_{1 N_{k}}^{y}, \ldots, a_{N 1}^{x}, a_{N 1}^{y}, a_{N 2}^{x}, a_{N 2}^{y}, \ldots, a_{N N_{k}}^{x}, a_{N N_{k}}^{y}\right]
$$

where $a_{i j}^{x}$ and $a_{i j}^{y}$ are the components along $\mathrm{x}$ and $\mathrm{y}$ of vector $\mathbf{a}_{i j}$ associated with node $i$ and enrichment function $\mathfrak{f}_{j}$. Matrix $\mathbf{F}$ is the $2 \times\left(2 N N_{k}\right)$ matrix

$$
\mathbf{F}=\left(\begin{array}{cccc}
1 & 2 & \ldots & N_{k} \\
\mathbf{F}_{1} & \mathbf{F}_{2} & \ldots & \mathbf{F}_{N_{k}}
\end{array}\right),
$$


638 being

$$
\mathbf{F}_{j 39}=\left(\begin{array}{ccccccc}
\mathfrak{f}_{j} & 0 & \mathfrak{f}_{j} & 0 & \ldots & \mathfrak{f}_{j} & 0 \\
0 & \mathfrak{f}_{j} & 0 & \mathfrak{f}_{j} & \ldots & 0 & \mathfrak{f}_{j}
\end{array}\right)
$$

${ }_{640}$ for $j=1, \ldots, N$. Hence, matrix $\mathbf{F}$ is a $2 \times\left(N N_{k}\right)$ matrix. Matrix $\mathbf{M}$ is a ${ }_{641}\left(2 N_{k} N\right) \times\left(2 N_{k} N\right)$ diagonal matrix

$$
\mathbf{M}=\begin{gathered}
1 \\
2 \\
\ldots 42 \\
N_{k}
\end{gathered}\left(\begin{array}{cccc}
1 & 2 & \ldots & N_{k} \\
\mathbf{M}_{M} & \mathbf{0} & \ldots & \mathbf{0} \\
\mathbf{0} & \mathbf{M}_{M} & \ldots & \mathbf{0} \\
\ldots & \ldots & \ldots & \mathbf{0} \\
\mathbf{0} & \mathbf{0} & \ldots & \mathbf{M}_{M}
\end{array}\right)
$$

${ }_{643}$ with $\mathbf{M}_{M}$ the $(2 N) \times(2 N)$ matrix

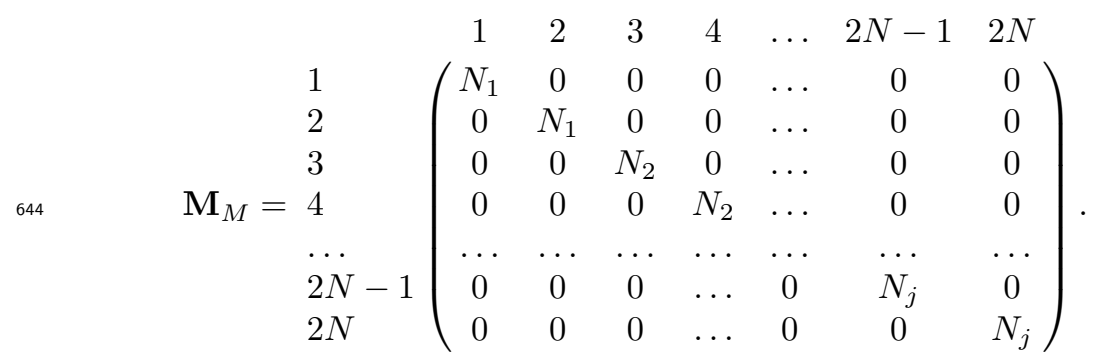

${ }_{645}$ Matrix $\tilde{\mathbf{F}}$ is the $3 \times\left(3 N_{k} N\right)$ matrix

$$
\begin{gathered}
\left.\quad \tilde{\mathbf{F}}=\begin{array}{cccccccc}
1 & 2 & 3 & \ldots & N \\
\tilde{F}_{1} & \tilde{\mathbf{F}}_{2} & \tilde{\mathbf{F}}_{3} & \ldots & \tilde{\mathbf{F}}_{N_{k}}
\end{array}\right), \\
{ }_{647} \text { with } \\
\tilde{\mathbf{F}}_{j}=\left(\begin{array}{cccccccccc}
\mathfrak{f}_{1} & 0 & 0 & \mathfrak{f}_{2} & 0 & 0 & \ldots & \mathfrak{f}_{N_{k}} & 0 & 0 \\
0 & \mathfrak{f}_{1} & 0 & 0 & \mathfrak{f}_{2} & 0 & \ldots & 0 & \mathfrak{f}_{N_{k}} & 0 \\
0 & 0 & \mathfrak{f}_{1} & 0 & 0 & \mathfrak{f}_{2} & \ldots & 0 & 0 & \mathfrak{f}_{N_{k}}
\end{array}\right), \\
{ }_{649} \text { for } j=1, \ldots, N . \text { Matrix } \mathbf{B}_{M} \text { is a }\left(3 N_{k} N\right) \times\left(2 N N_{k}\right) \text { that reads }
\end{gathered}
$$

650

$$
\mathbf{B}_{M}=\begin{gathered}
1 \\
2 \\
\ldots \\
N
\end{gathered}\left(\begin{array}{cccc}
\mathbf{B}_{M 1} & \mathbf{0} & \mathbf{0} & \mathbf{0} \\
\mathbf{0} & \mathbf{B}_{M 2} & \mathbf{0} & \mathbf{0} \\
\cdots & \cdots & \cdots & \cdots \\
\mathbf{0} & \mathbf{0} & \mathbf{0} & \mathbf{B}_{M N}
\end{array}\right)
$$


where $\mathbf{B}_{M j}$ is the compatibility matrix

$$
\mathbf{B}_{M j}=\begin{gathered}
1 \\
1 \\
2 \\
\ldots \\
N_{k}
\end{gathered}\left(\begin{array}{ccccc}
\mathbf{B}_{j} & \mathbf{0} & \mathbf{0} & \ldots & \mathbf{0} \\
\mathbf{0} & \mathbf{B}_{j} & \mathbf{0} & \ldots & \mathbf{0} \\
\ldots & \ldots & \ldots & \ldots & \ldots \\
\mathbf{0} & \mathbf{0} & \mathbf{0} & \ldots & \mathbf{B}_{j}
\end{array}\right)
$$

and

$$
\mathbf{B}_{j}=\left(\begin{array}{cc}
N_{j, x} & 0 \\
0 & N_{j, y} \\
N_{j, y} & N_{j, x}
\end{array}\right)
$$

for $j=1, \ldots, N$.

\section{References}

[1] N. Moës, J. Dolbow, T. Belytschko, A finite element method for crack growth without remeshing, Int. J. Num. Meth. Engng 46 (1999) 131-150.

[2] N. Sukumar, D. Chopp, N. Moës, T. Belytschko, Modeling holes and inclusions by level sets in the extended finite-element method, Comp. Meth. Appl. Mech. Engng. 190 (2001) 6183-6200.

[3] N. Moës, T. Belytschko, Extended finite element method for cohesive crack growth, Engng. Fract. Mech. 69 (2002) 813-833.

[4] T. Belytschko, R. Gracie, G. Ventura, A review of the extended/generalized finite element methods for material modelling, Modelling and Simulation in Materials Science and Engineering 17, DOI: 10.1088/09650393/17/4/043001. doi:10.1088/0965-0393/17/4/043001.

[5] J. Melenk, I. Babuska, The partition of unity finite element method: Basic theory and applications, Comp. Meth. Appl. Mech. Engng. 139 (1996) 289314.

[6] C. Duarte, I. Babuska, J. Oden, Generalized finite element methods for three- dimensional structural mechanics problems, Comp. Struct. 77 (2000) $215-232$.

[7] X. Liu, Q. Xiao, B. Karihaloo, XFEM for direct evaluation of mixed mode SIFs in homogeneous and bi-materials, Int. J. Num. Meth. Engng 59 (2004) 1103-1118.

[8] M. Lan, H. Waisman, I. Harari, A high-order extended finite element method for extraction of mixed-mode strain energy release rates in arbitrary crack settings based on irwins integral, Int. J. Num. Meth. Engng 96 (2013) 787-812. 
[9] J. Chessa, H. Wang, T. Belytschko, On the construction of blending elements for local partition of unity enriched finite elements, Int. J. Num. Meth. Engng 57 (2003) 1015-1038.

[10] P. Laborde, J. Pommier, Y. Renard, M. Salaün, Analysis of stresses and strains near the end of a crack traversing a plate, Int. J. Num. Meth. Engng 64 (2005) 354-381.

[11] T. Fries, A corrected XFEM approximation without problems in blending elements, Int. J. Num. Meth. Engng 75 (2007) 503-532.

[12] G. Ventura, R. Gracie, T. Belytschko, Fast integration and weight function blending in the extended finite element method, Int. J. Num. Meth. Engng 77 (2009) 1-29.

[13] I. Babuška, U. Banerjee, Stable generalized finite element method (sgfem), Comp. Meth. Appl. Mech. Engng. (2012) 91-111.

[14] S. Mousavi, J. Pask, N. Sukumar, Efficient adaptive integration of functions with sharp gradients and cusps in n-dimensional parallelepipeds, Int. J. Num. Meth. Engng 91 (2012) 343-357.

[15] V. Gupta, C. Duarte, I. Babuška, U. Banerjee, A stable and optimally convergent generalized FEM (SGFEM) for linear elastic fracture mechanics, Comp. Meth. Appl. Mech. Engng. 266 (2013) 23-39.

[16] V. Gupta, C. Duarte, I. Babuška, U. Banerjee, Stable GFEM (SGFEM): Improved conditioning and accuracy of GFEM/XFEM for threedimensional fracture mechanics, Comp. Meth. Appl. Mech. Engng. 289 (2013) 355-386.

[17] T. Fries, T. Belytschko, The intrinsic XFEM: a method for arbitrary discontinuities without additional unknowns, Int. J. Num. Meth. Engng 68 (2006) 1358-1385.

[18] S. Löhnert, A stabilization technique for the regularization of nearly singular extended finite elements, Comp. Mech. 54 (2014) 523-533.

[19] J. Wu, F. Li, An improved stable XFEM (is-XFEM) with a novel enrichment function for the computational modeling of cohesive cracks, Comp. Meth. Appl. Mech. Engng. 295 (2015) 77-107.

[20] R. Alicandro, A. Braides, J. Shah, Free-discontinuity problems via functionals involving the L1-norm of the gradient and their approximation, Interfaces and Free Boundaries 1 (1999) 17-37.

[21] L. Ambrosio, N. Fusco, D. Pallara, Functions of Bounded Variation and Free Discontinuity Problems, Oxford Mathematical Monographs, 2000.

[22] B. Bourdin, G. Francfort, J.-J. Marigo, Numerical experiments in revisited brittle fracture, J. Mech. Phys. Sol. 48 (2000) 797-826. 
[23] R. Gracie, H. Wang, T. Belytschko, Blending in the extended finite element method by discontinuous galerkin and assumed strain methods, Int. J. Num. Meth. Engng 74 (2008) 1645-1669.

[24] V. Volterra, Sur l'équilibre des corps élastiques multiplement connexes, Annales scientifiques de l'Ecole Normale superieure 24 (1907) 401-517.

[25] C. Somigliana, Sulla teoria delle distorsioni elastiche, Rendiconti dei Lincei XXIV (1915) 655-666.

[26] H. Reißner, Eigenspannungen und Eigenspannungsquellen, Zeit. Angew. Math. Mech. 11 (1931) 1-8.

[27] G. Colonetti, Per una teoria generale delle coazioni elastiche, Atti R. Acad. Sci. Torino: Cl. Sci. Fis. Mat. Natur. 56 (1921) 188-198.

[28] J. Eshelby, The force on elastic singularity, Philosophical Transactions of the Royal Society of London A: Mathematical and Physical Sciences 244 (1951) 87-112.

[29] T. Mura, Micromechanics of Defects in Solids., Martinus Nijhof, The Hague, The Netherlands, 1982.

[30] T. Mura, R. Furuhashi, The elastic inclusion with a sliding interface, J.Appl. Mech. 51 (1984) 308-310.

[31] B. Schmidt, F. Fraternali, M. Ortiz, Eigenfracture: An eigendeformation approach to variational fracture, Multiscale Modeling and Simulations 7 (2009) 12371266.

[32] E. Benvenuti, A regularized XFEM framework for embedded cohesive interfaces, Comp. Meth. Appl. Mech. Engng. 197 (2008) 4367-4378.

[33] E. Benvenuti, A. Tralli, Simulation of finite-width process zone for concretelike materials, Comp. Mech. 50 (2012) 479-497.

[34] E. Benvenuti, G. Ventura, N. Ponara, A.Tralli, Variationally consistent eXtended FE model for 3D planar and curved imperfect interfaces, Comp. Meth. Appl. Mech. Engng. 267 (2013) 1-22.

[35] E. Benvenuti, XFEM with equivalent eigenstrain for matrixinclusion interfaces, Comp. Mech. 53 (2014) 893-908.

[36] J. Eshelby, The elastic energy-momentum tensor, J. Elasticity 5 (1975) 321-335.

[37] J. Eshelby, The determination of the elastic field of an ellipsoidal inclusion, and related problems, Proc. Royal Society A: Mathematical, Physical and Engineering Sciences 241 (1957) 376-396. 
[38] M. Kanninen, C. Popelar, Advanced Fracture Mechanics, Oxford University Press, 1985.

[39] H. Westergaard, Bearing pressures and cracks, Journal of Applied Mechanics 6 (1939) A49-53.

[40] G. Irwin, Analysis of stresses and strains near the end of a crack traversing a plate, ASME Applied Mechanics no. 57 APM-22 (1956) 361-364.

[41] J. Eshelby, Fracture mechanics, Sci. Prog. Oxford 59 (1971) 161-179.

[42] J. Eshelby, The elastic field outside an ellipsoidal inclusion, Proc. Royal Society A: Mathematical, Physical and Engineering Science 252 (1959) 561569.

[43] A. Love, A Treatise on the Mathematical Theory of Elasticity, Cambridge University Press, 1927.

[44] E. Noether, Nachrichten von der Gesellschaft der Wissenschaften zu Göttingen, Mathematisch-Physikalische Klasse (1918) 235-257.

[45] J. Rice, A path independent integral and the approximate analysis of strain concentration by notches and cracks, J. Appl. Mech. 35 (1968) 379-386.

[46] J. Tarancón, A. Vercher, E. Giner, F. Fuenmayor, Enhanced blending elements for XFEM applied to linear elastic fracture mechanics, Int. J. Num. Meth. Engng 77 (2009) 126-148.

[47] K. Cheng, T.-P. Fries, Higher-order XFEM for curved strong and weak discontinuities, Int. J. Num. Meth. Engng 82 (2010) 564-590.

[48] E. Benvenuti, A. Tralli, G. Ventura, A regularized xfem model for the transition from continuous to discontinuous displacements, Int. J. Num. Meth. Engng 197 (2008) 4367-4378.

[49] N. Sukumar, J. Dolbow, N. Moës, Extended finite element method in computational fracture mechanics: a retrospective examination, Int. J. Fract. 196 (2015) 189-206.

[50] G. Song, H. Waisman, M. Lan, I. Harari, Extraction of stress intensity factors from irwins integral using high-order XFEM on triangular meshes, Int. J. Num. Meth. Engng 102 (2012) 528-550.

[51] H.Tada, P. Paris, G. Irwin, The Stress Analysis of Cracks Handbook, Third Edition, ASME Press, US, 2000.

[52] F. Stazi, E. Budyn, J. Chessa, T. Belytschko, An extended finite element method with higher-order elements for curved cracks, Comp. Mech. 31 (2003) 38-48. 


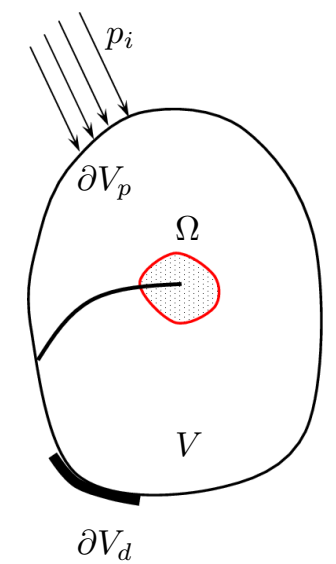

Figure 1: Body with a crack

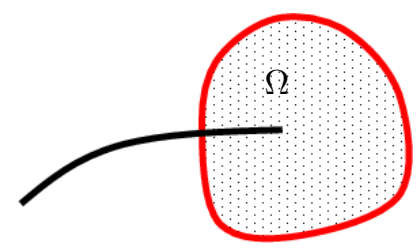

Figure 2: Region $\Omega$ of the elastic singularity 


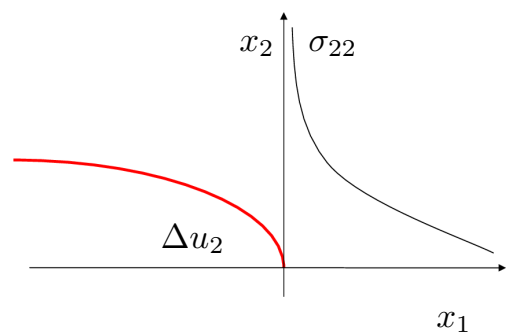

Figure 3: Mode I opening and stress singularity at the crack tip

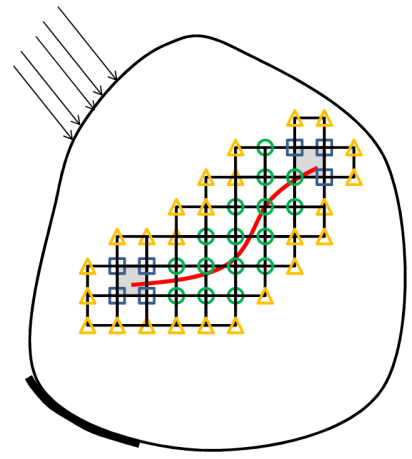

Figure 4: Discretization of the domain according to the enriched formulation 


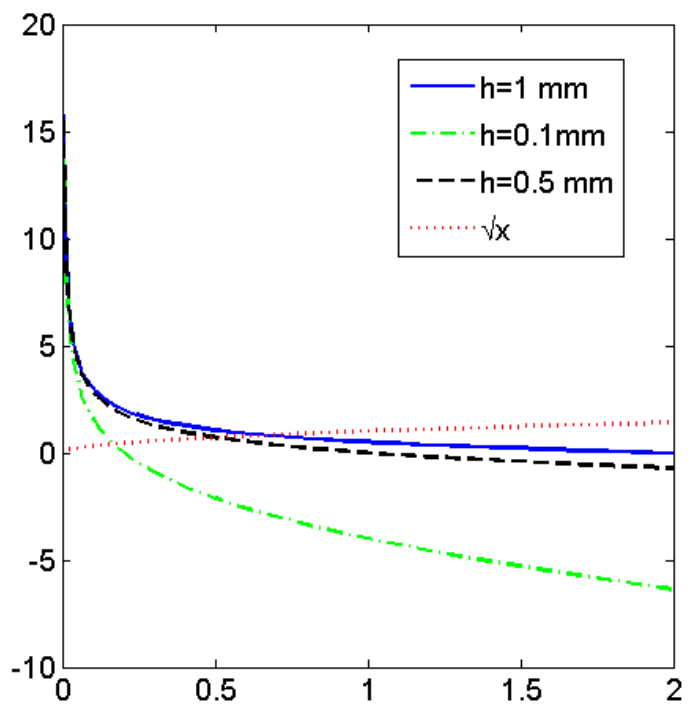

Figure 5: Comparison between the $\sqrt{x}$-term (red dotted line) and function $\left(1-\frac{\bar{x}}{h}\right) \frac{1}{2 \sqrt{x}}$ for variable mesh size $h$

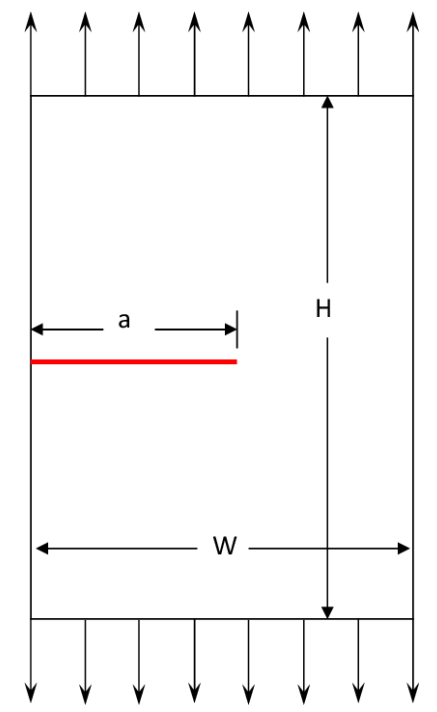

Figure 6: Single Edge Notched test (SEN) 


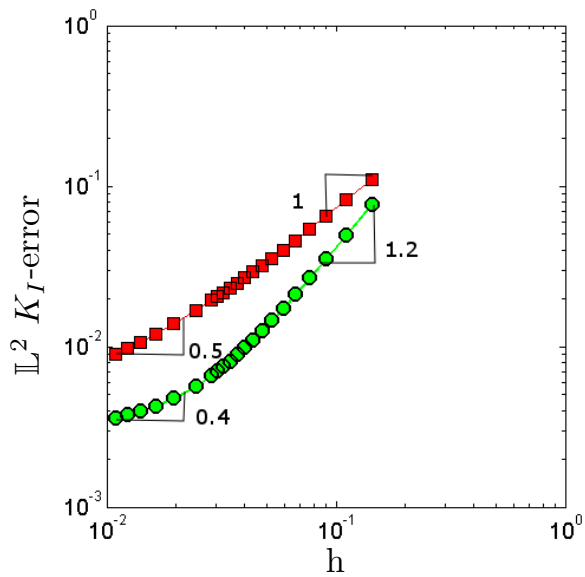

(a)

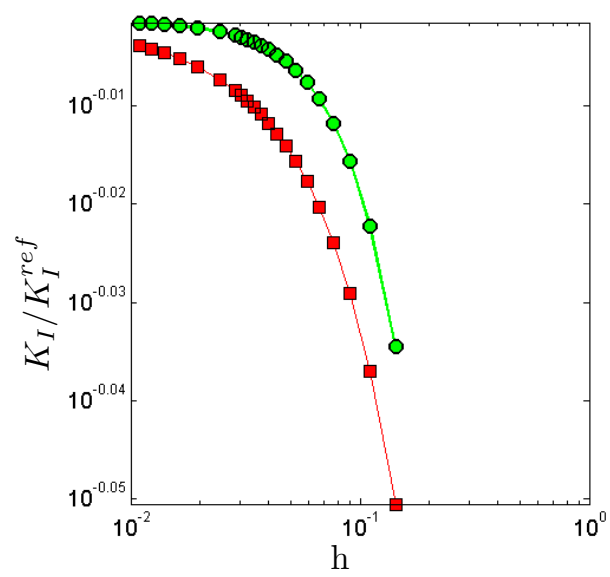

(b)

Figure 7: SEN: relative error in normalized discrete $\mathbb{L}^{2}$ norm of $K_{I}$ (a) and ratio $K_{I} / K_{I}^{\text {ref }}$ (b) for variable mesh size with $a=2 W / 3$. The red squares denote the results obtained by means of the standard XFEM, while the green circles denote those obtained by means of the present XFEM. 


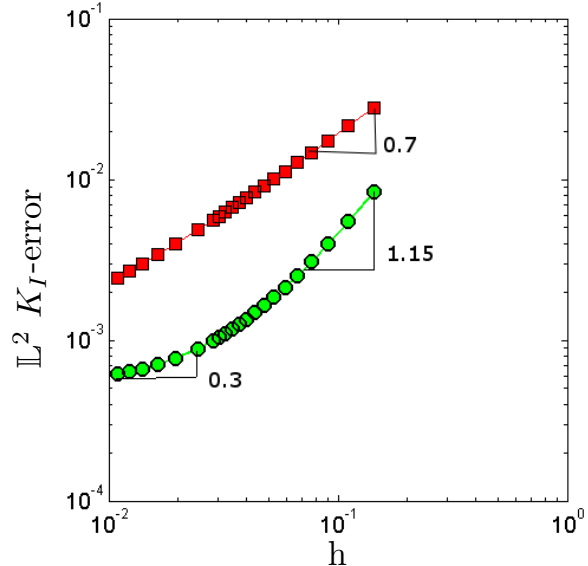

(a)

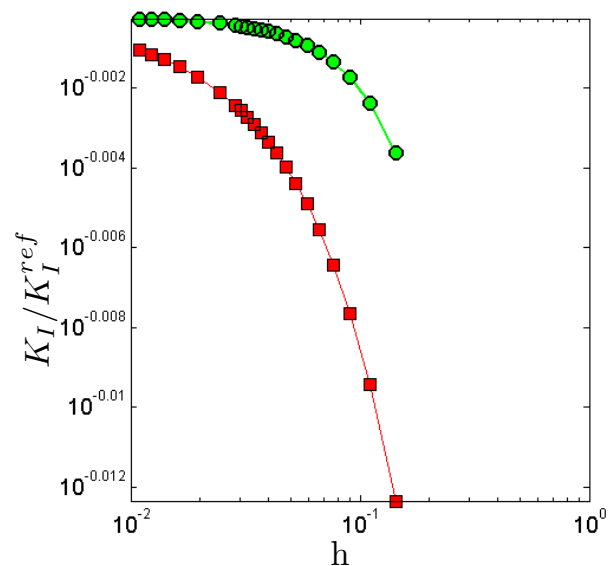

(b)

Figure 8: SEN: relative error in normalized discrete $\mathbb{L}^{2}$ norm of $K_{I}$ (a) and ratio $K_{I} / K_{I}^{\text {ref }}$ (b) for variable mesh size with $a=W / 2$. The red squares denote the results obtained by means of the standard XFEM, while the green circles denote those obtained by means of the present XFEM

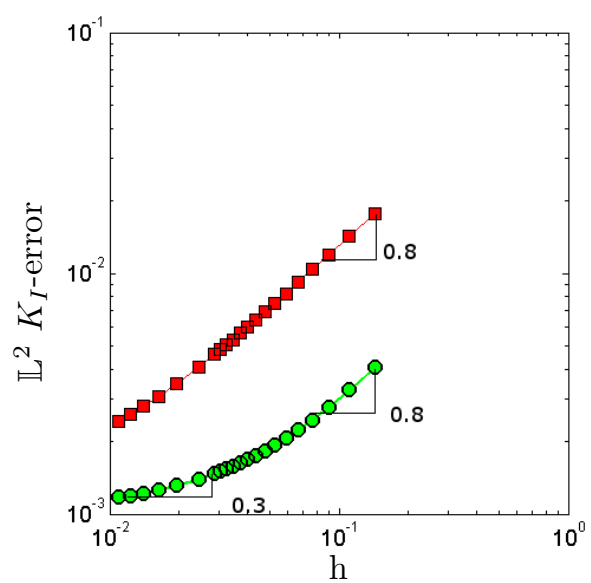

(a)

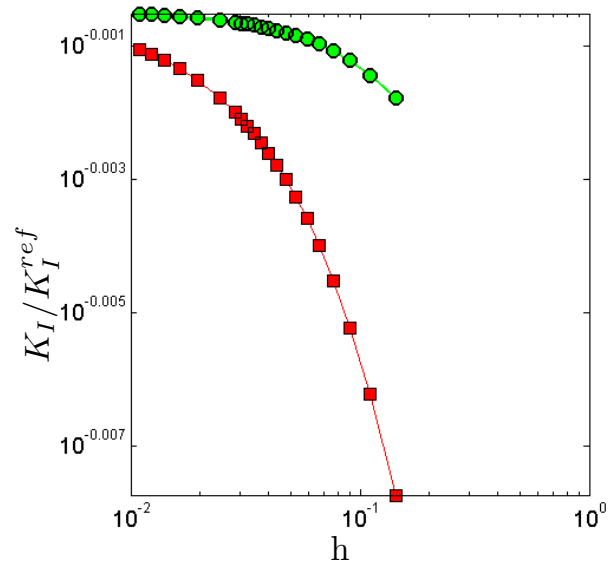

(b)

Figure 9: SEN: relative error in normalized discrete $\mathbb{L}^{2}$ norm of $K_{I}$ (a) and ratio $K_{I} / K_{I}^{\text {ref }}$ (b) for variable mesh size with $a=W / 6$; red squares and green circles denote the results obtained by means of the standard XFEM and the present XFEM, respectively 


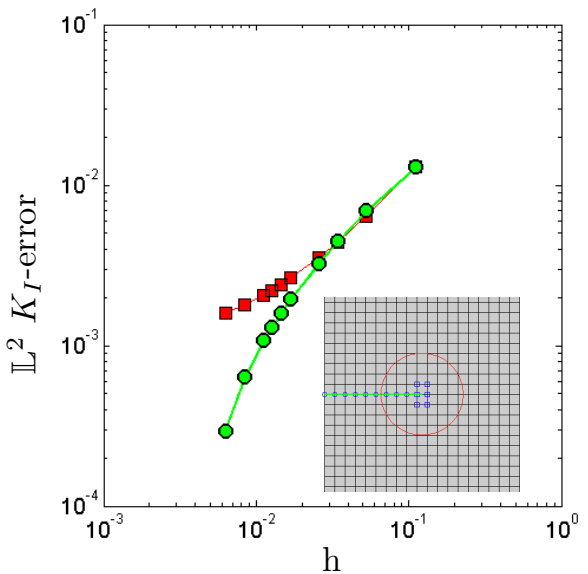

(a)

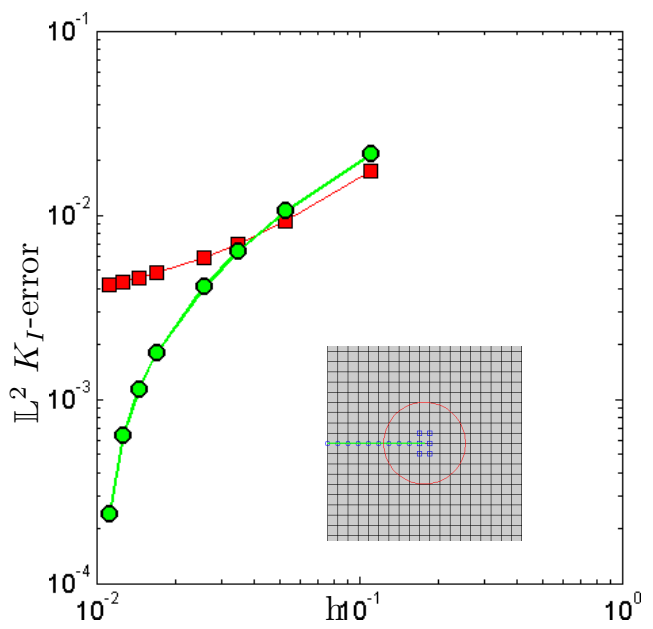

(b)

Figure 10: SEN with crack aligned along the element edges: relative error in normalized discrete $\mathbb{L}^{2}$ norm of $K_{I}$ for variable mesh size with $a=0.5 \mathrm{~W}$ (a) and $a=0.6 \mathrm{~W}$; green circles and red squares denote the present formulation and the standard XFEM, respectively

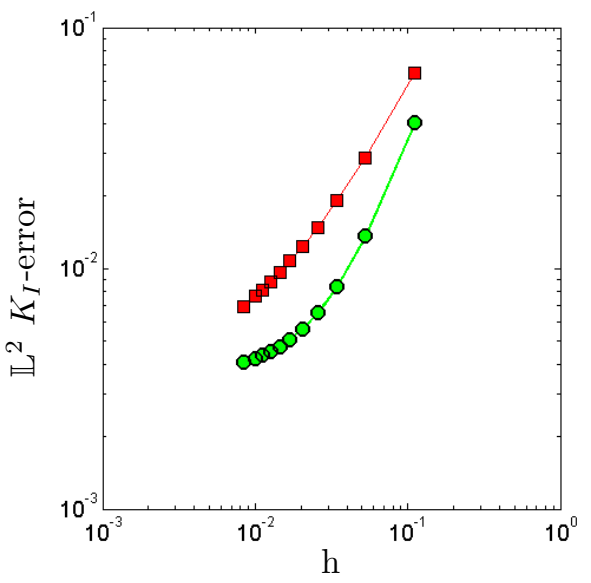

(a)

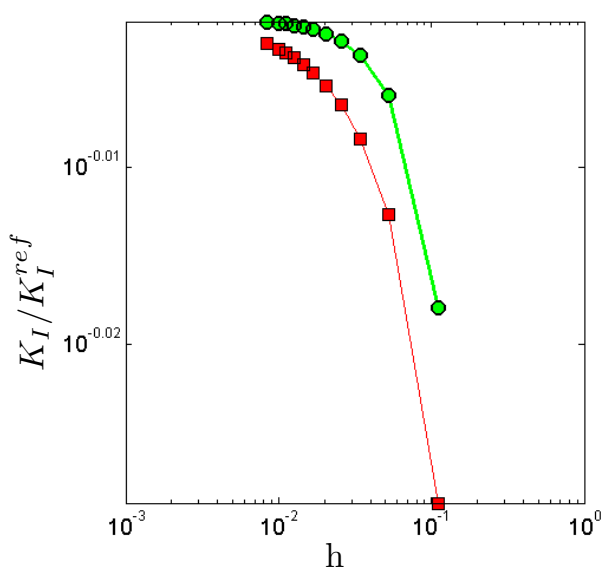

(b)

Figure 11: SEN with random crack tip position with respect to the finite element: relative error in normalized discrete $\mathbb{L}^{2}$ norm of $K_{I}$ and ratio $K_{I} / K_{I}^{r e f}$ (b) for variable mesh size with $a=0.8 \mathrm{~W}$; green circles and red squares denote the present formulation and the standard XFEM, respectively 


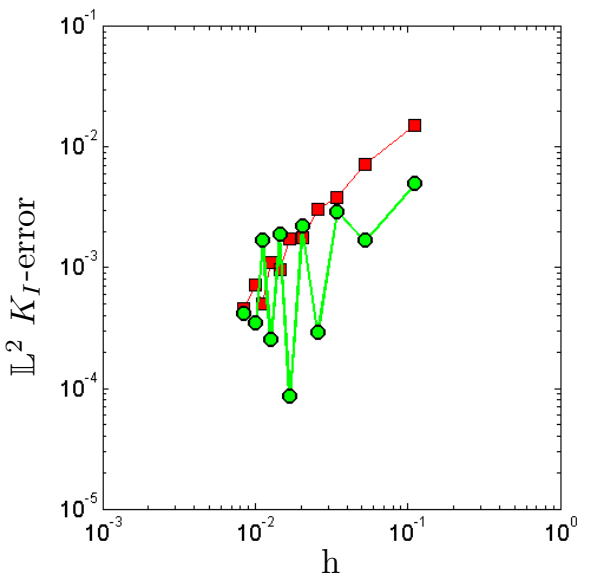

(a)

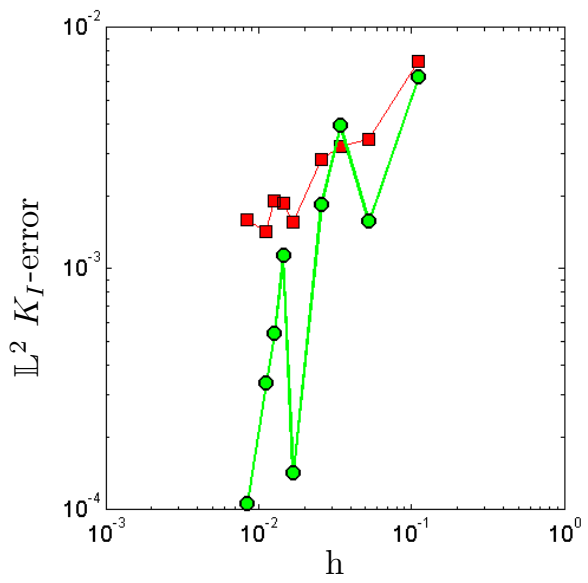

(b)

Figure 12: SEN with random crack tip position with respect to the finite element: relative error in normalized discrete $\mathbb{L}^{2}$ norm of $K_{I}$ (a) and ratio $K_{I} / K_{I}^{r e f}$ (b) for variable mesh size with $a=0.45 W$ (a) and $a=0.175 W$ (b); green circles and red squares denote the present formulation and the standard XFEM, respectively 


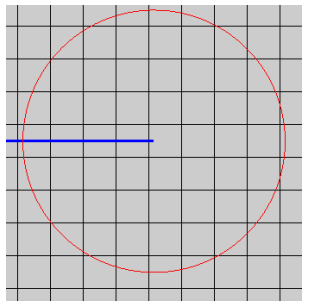

(a) $\mathrm{h}=1 / 9 \mathrm{~mm}$
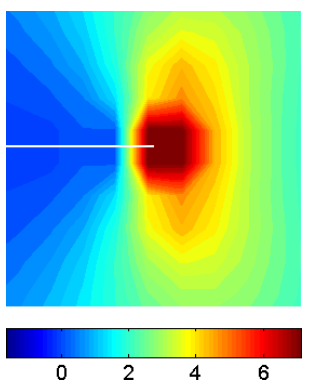

(d) present XFEM, $\mathrm{h}=1 / 9 \mathrm{~mm}, K_{I}=5.01$
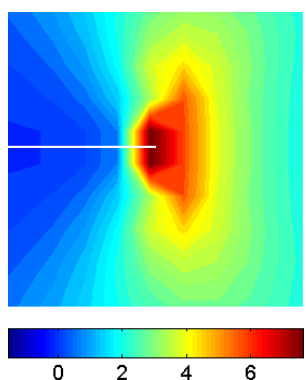

(g) standard XFEM, $\mathrm{h}=1 / 9 \mathrm{~mm}, K_{I}=4.91$

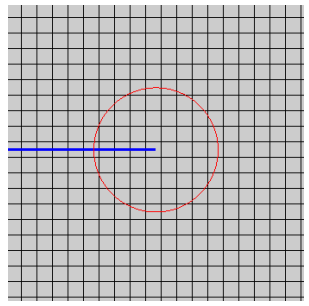

(b) $\mathrm{h}=1 / 19 \mathrm{~mm}$
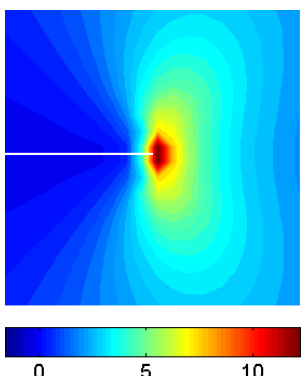

(e) present XFEM,

$\mathrm{h}=1 / 19$

$K_{I}=4.98$

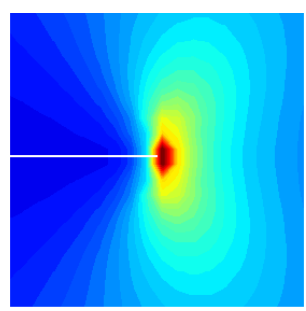

10

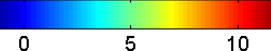

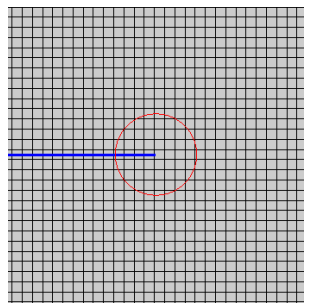

(c) $\mathrm{h}=1 / 29 \mathrm{~mm}$
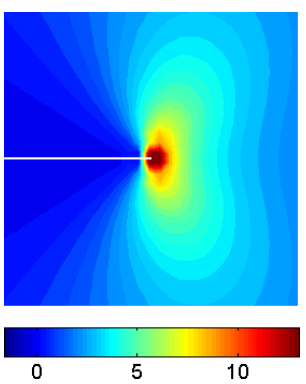

(f) present XFEM

$\mathrm{mm}, \quad \mathrm{h}=1 / 29$

$K_{I}=5.00$

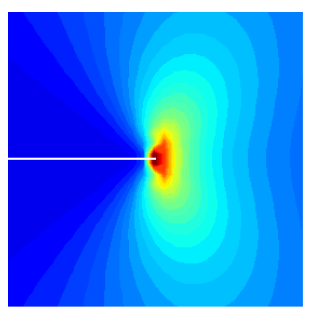

(h) standard XFEM, $\mathrm{h}=1 / 19 \mathrm{~mm}, K_{I}=$ 4.95

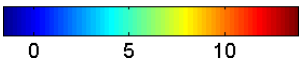

(i) standard XFEM, $\mathrm{h}=1 / 29$ $K_{I}=4.97$

Figure 13: SEN with random crack tip position with respect to the finite element: comparison between $\sigma_{22}$ plotted for variable mesh size $h$; the reference value is $K_{I}^{r e f}=4.99$ with a $0.5 \%$ of precision 51 . 

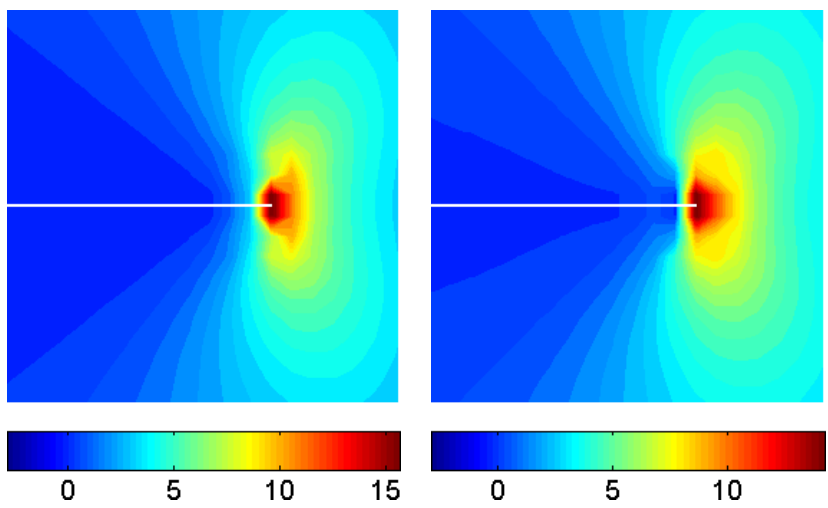

(a) $\sigma_{22}$, Standard XFEM

(b) $\sigma_{22}$, Present XFEM
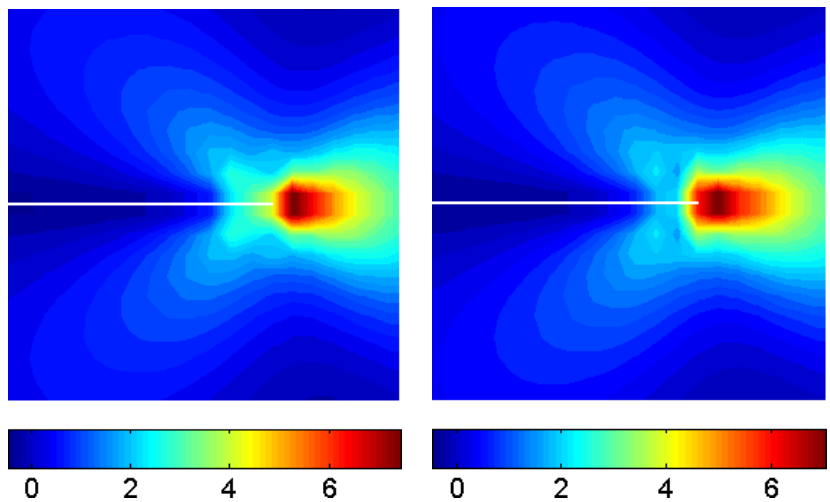

(c) $\sigma_{11}$, Standard XFEM

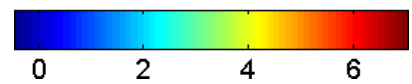

(d) $\sigma_{11}$, Present XFEM
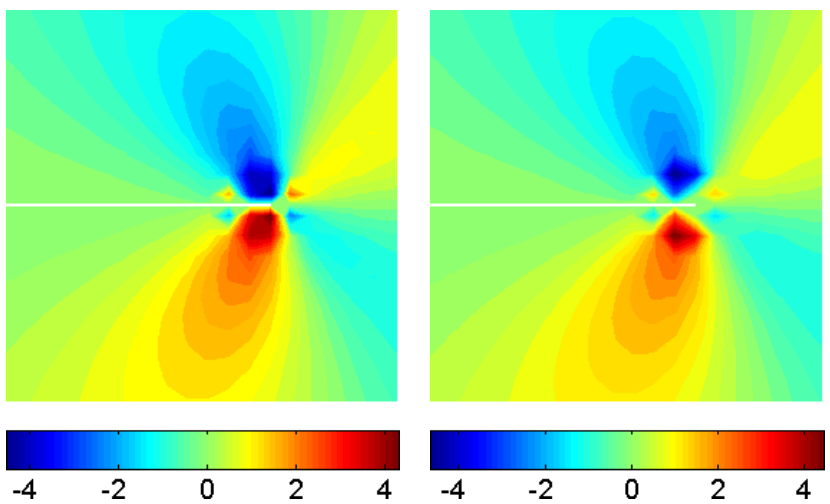

(e) $\sigma_{12}$, Standard XFEM

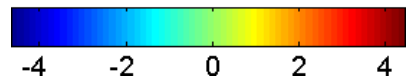

(f) $\sigma_{12}$, Present XFEM

Figure 14: SEN: Comparison between the contour plots of $\sigma_{12}[\mathrm{MPa}]$ obtained for $a=0.5 \mathrm{~W}$ and 57x114 elements. 


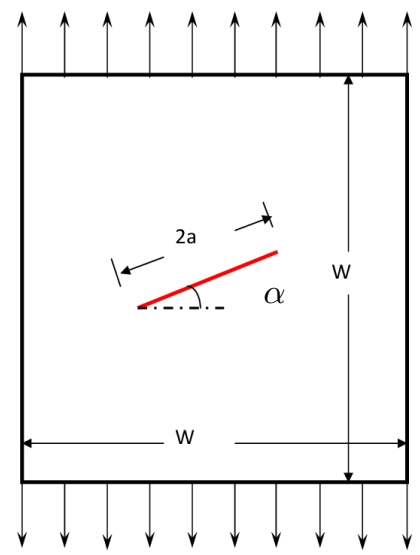

Figure 15: CCT: Central inclined crack in a square plate of edge length $10 \mathrm{~mm}$ with crack length $2 a$.

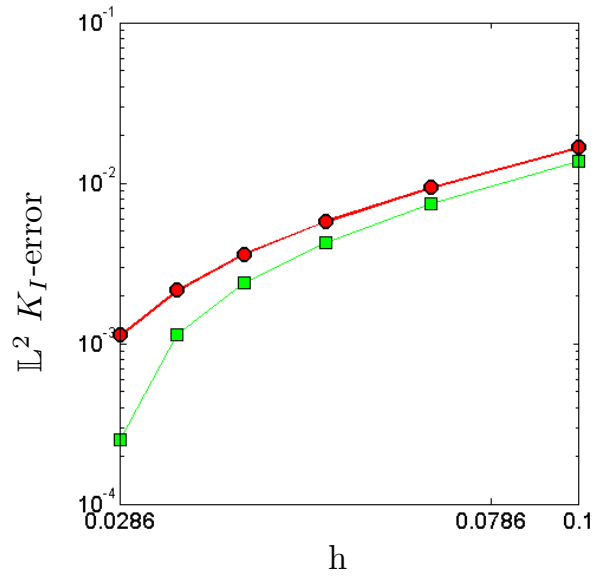

(a)

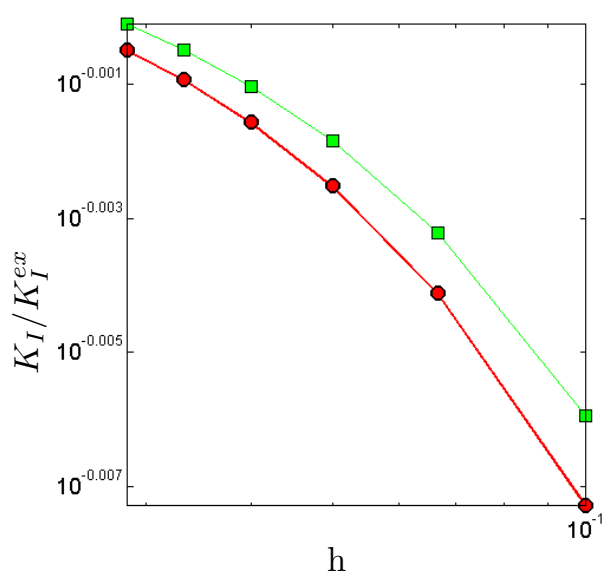

(b)

Figure 16: CCT with horizontal crack: relative error in normalized discrete $\mathbb{L}^{2}$ norm of $K_{I}$ (a) and ratio $K_{I} / K_{e x}$ (b) for variable mesh size and $2 a=8 \mathrm{~W} / 100$; green squares and red circles denote the proposed XFEM and the standard XFEM, respectively 


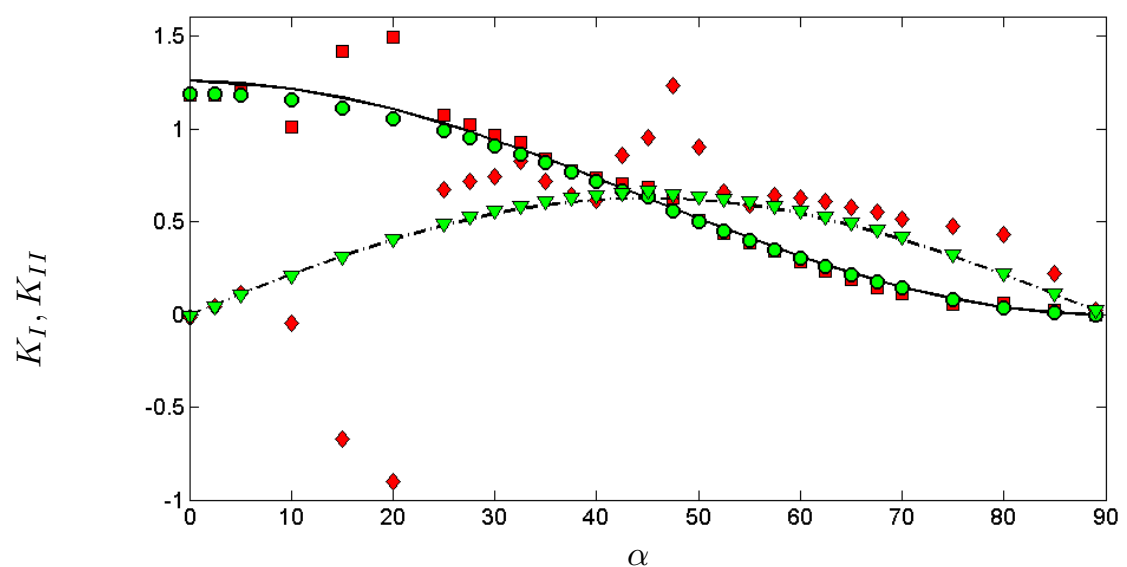

(a)

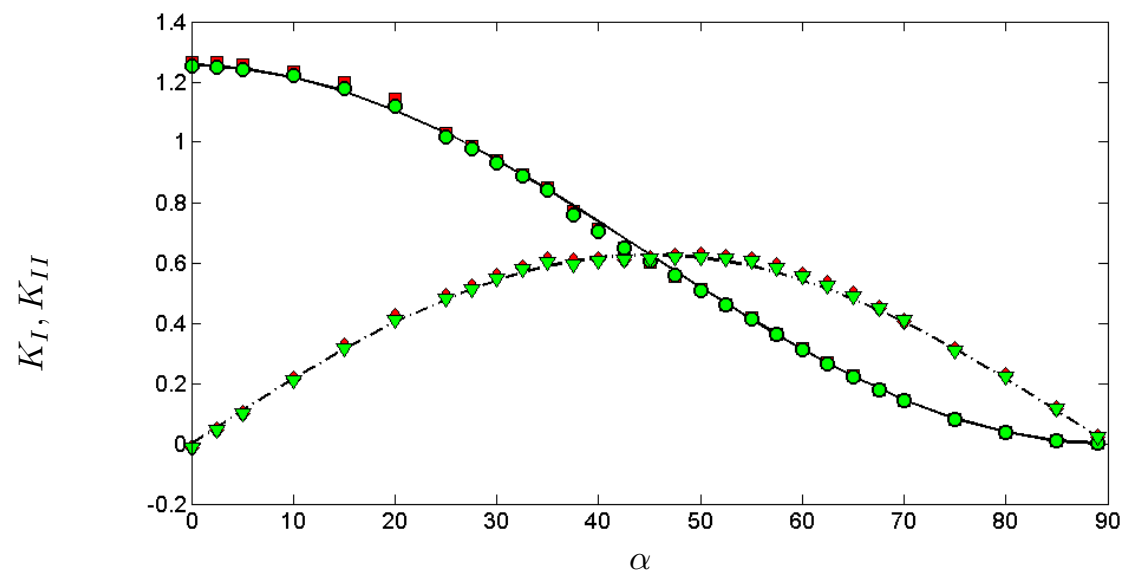

(b)

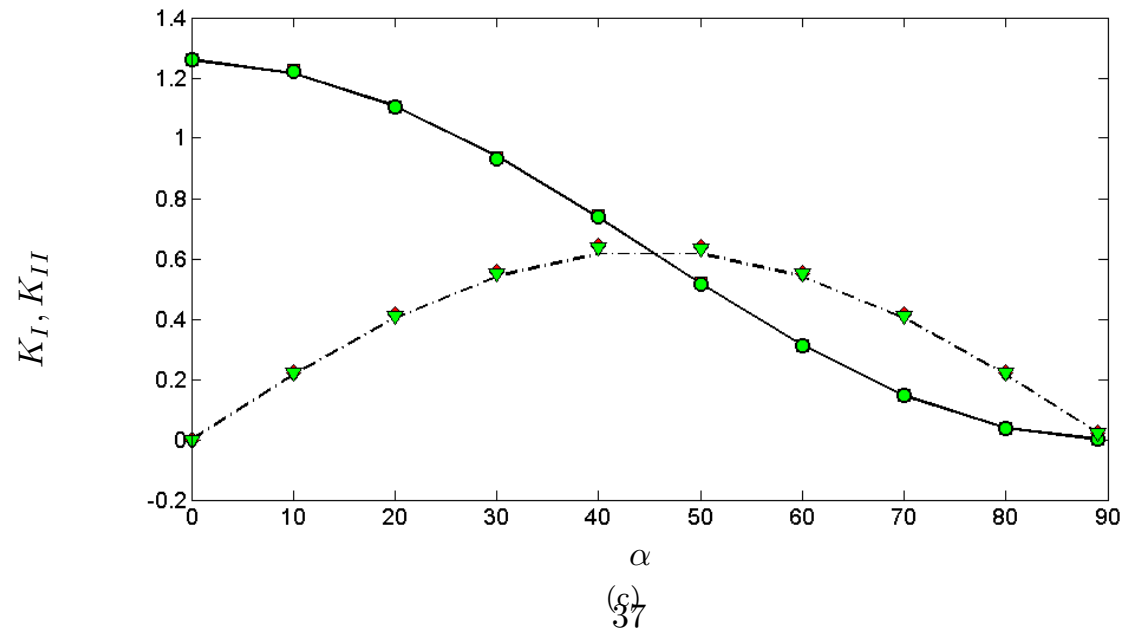

Figure 17: CCT: $K_{I}$ and $K_{I I}$ obtained with meshes of 49x49 (a), 99x99 (b), and 199 x 199 (c) finite elements for variable inclination $\alpha$ (rad) and crack length $2 a=W / 10$; green circles and triangles denote the proposed XFEM, red squares and diamonds denote the standard XFEM 


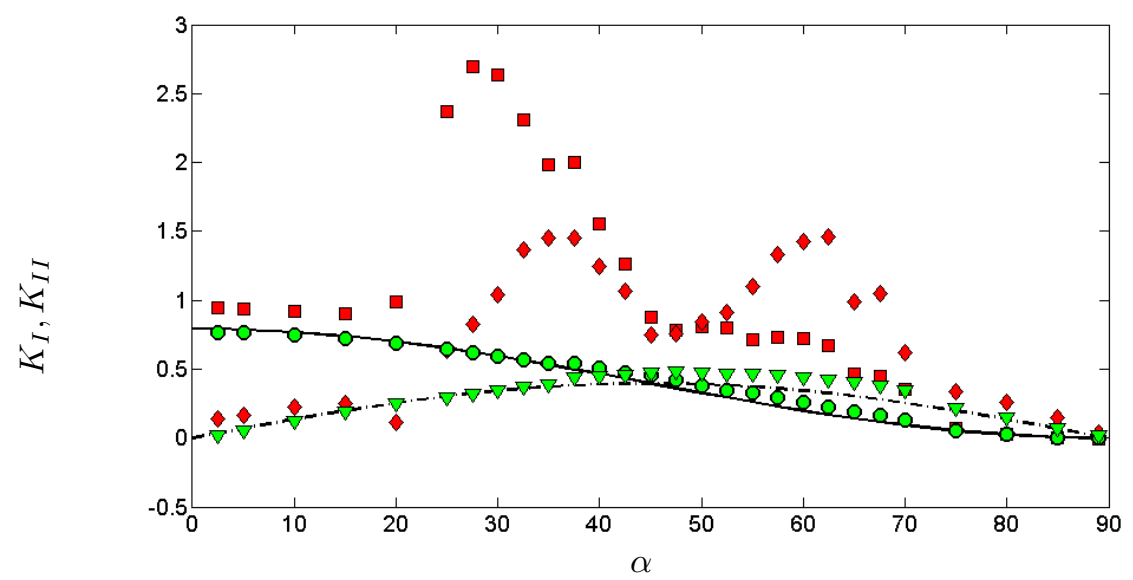

(a)

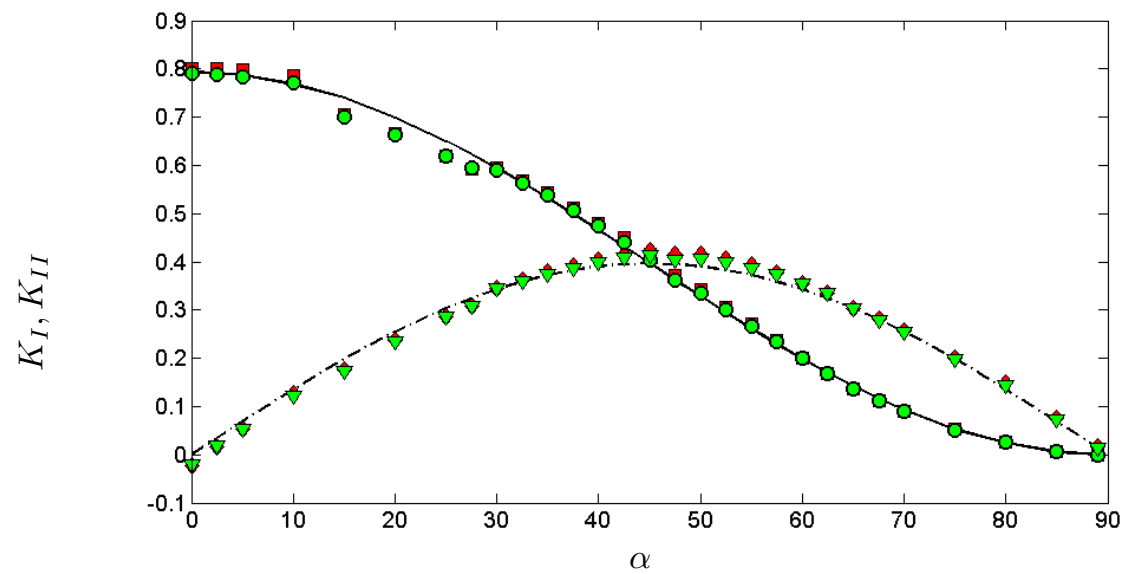

(b)

Figure 18: CCT: $K_{I}$ and $K_{I I}$ obtained with $99 \times 99$ (a) and 199 x 199 (b) finite elements for variable inclination $\alpha$ (rad) and crack length $2 a=W / 25$; green circles and triangles denote the proposed XFEM, red squares and diamonds denote the standard XFEM 


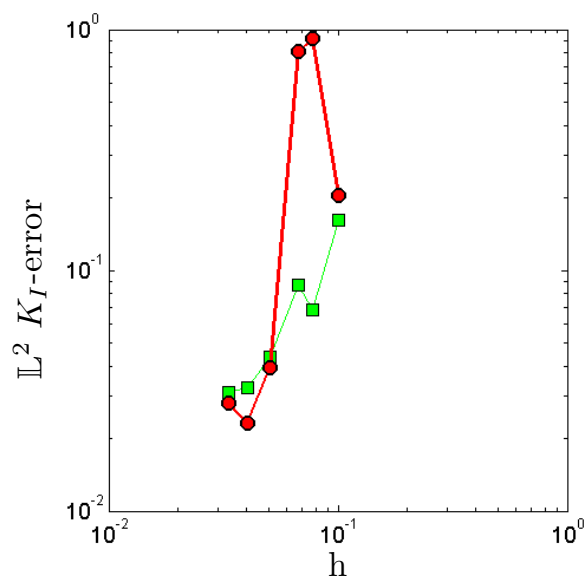

(a)

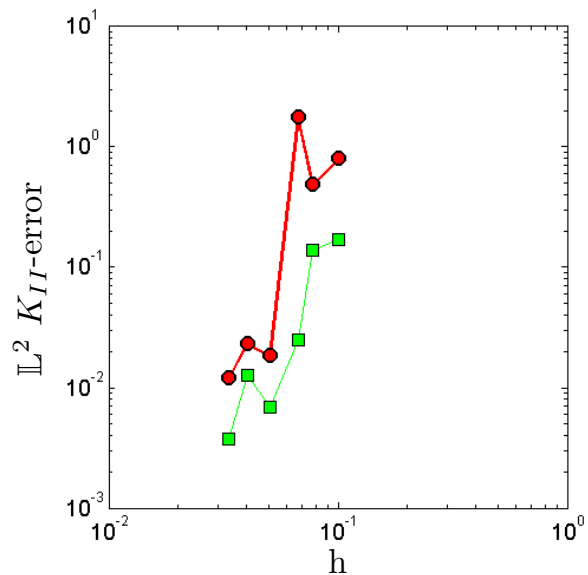

(c)

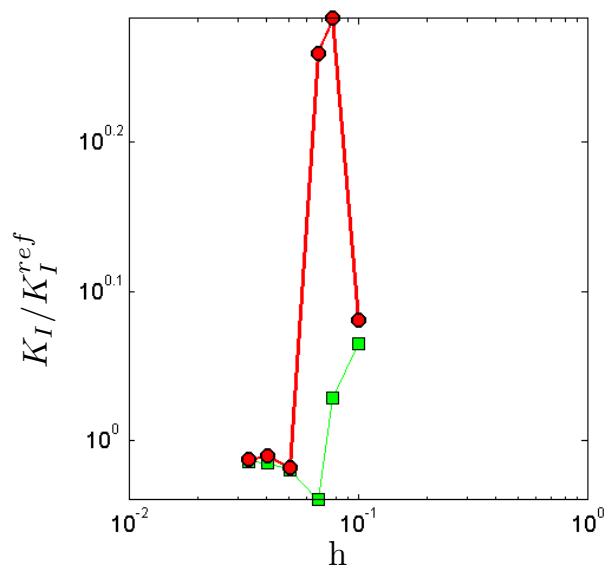

(b)

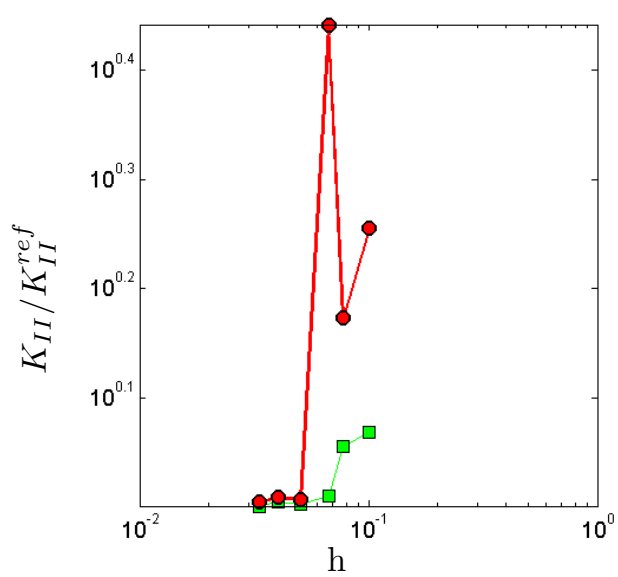

(d)

Figure 19: CCT: relative error in normalized discrete $\mathbb{L}^{2}$ norm of $K_{I}$ (a), $K_{I I}$ (c) and ratio $K_{I} / K_{I}^{r e f}(\mathrm{~b})$ and $K_{I I} / K_{I I, e x}(\mathrm{~d})$ for variable mesh size with fixed crack length $2 a=3 \mathrm{~W} / 100$ and $\alpha=\pi / 4$; green squares and red circles denote the proposed XFEM and the standard XFEM, respectively. 

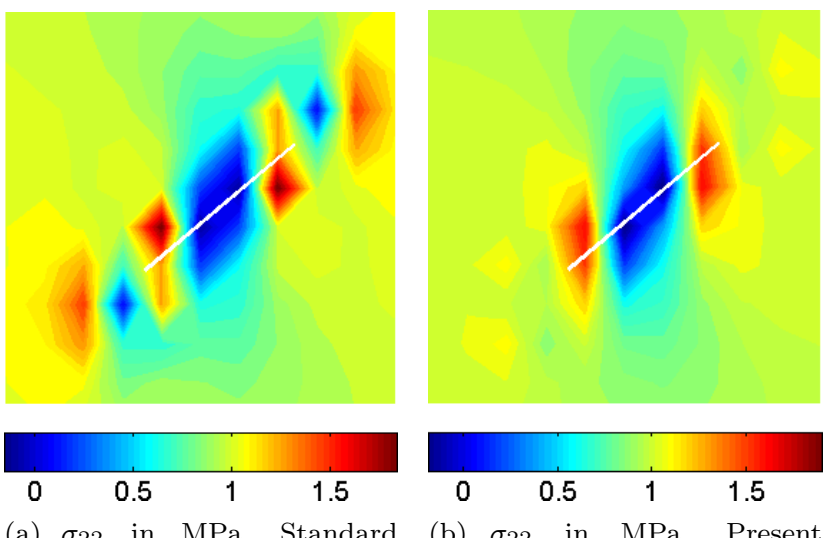

(a) $\sigma_{22}$ in $\mathrm{MPa}, \quad$ Standard XFEM

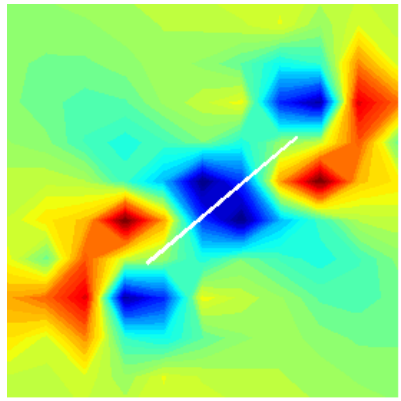

(b) $\sigma_{22}$ XFEM
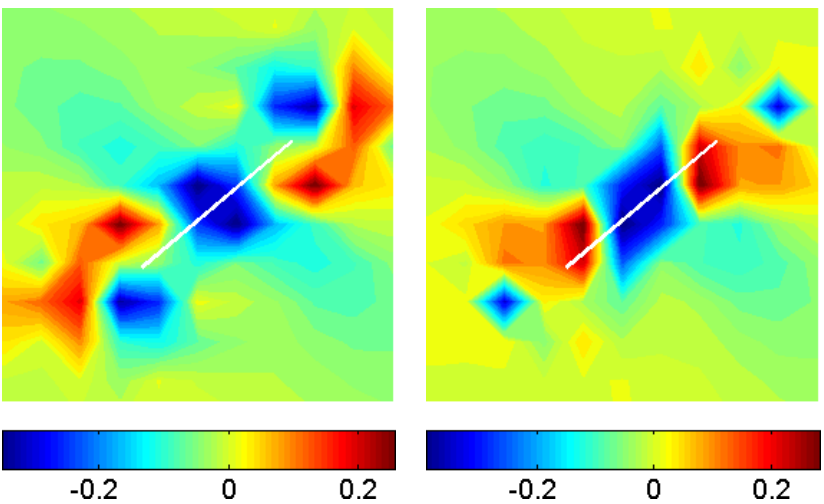

(c) $\sigma_{11}$ in $\mathrm{MPa}$, Standard XFEM

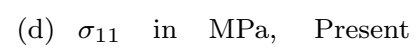
XFEM
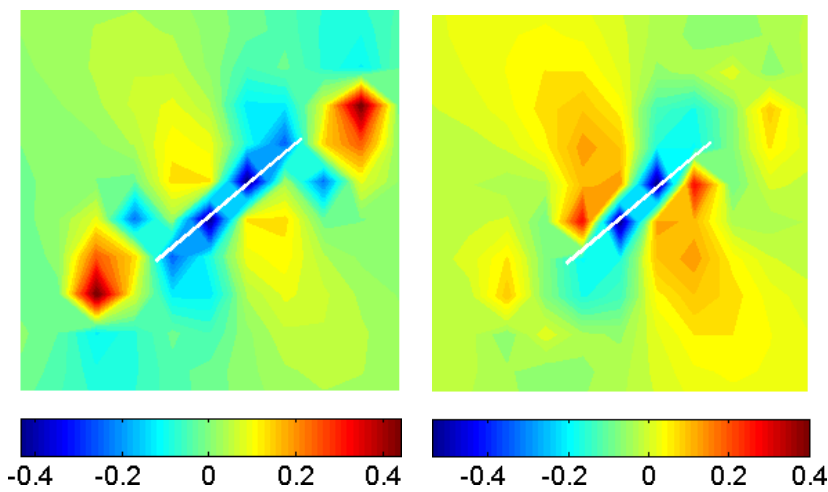

(e) $\sigma_{12}$ in $\mathrm{MPa}$, Standard (f) $\sigma_{12}$ in $\mathrm{MPa}$, Present XFEM XFEM

Figure 20: CCT: contour plots of $\sigma_{22}, \sigma_{11}$, and $\sigma_{12}$ for $\alpha=40^{\circ}, 2 a=W / 10$, and $49 \times 49$ finite elements. 

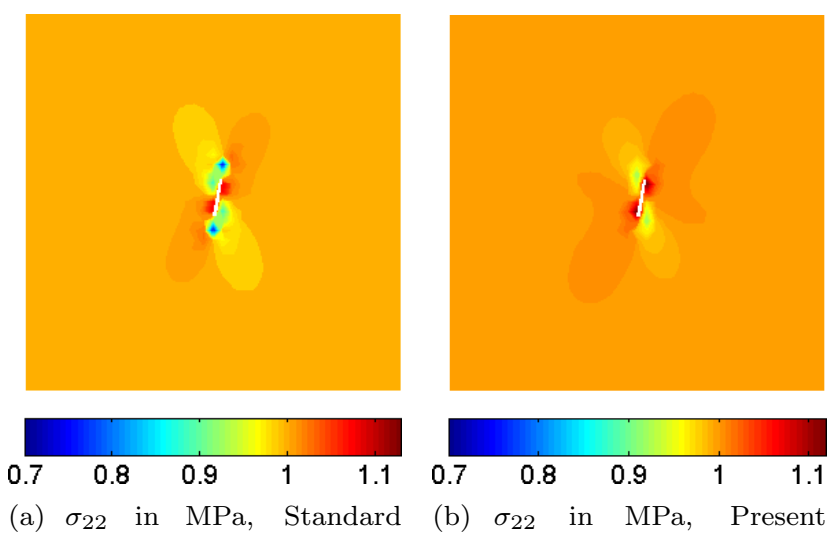
XFEM

XFEM
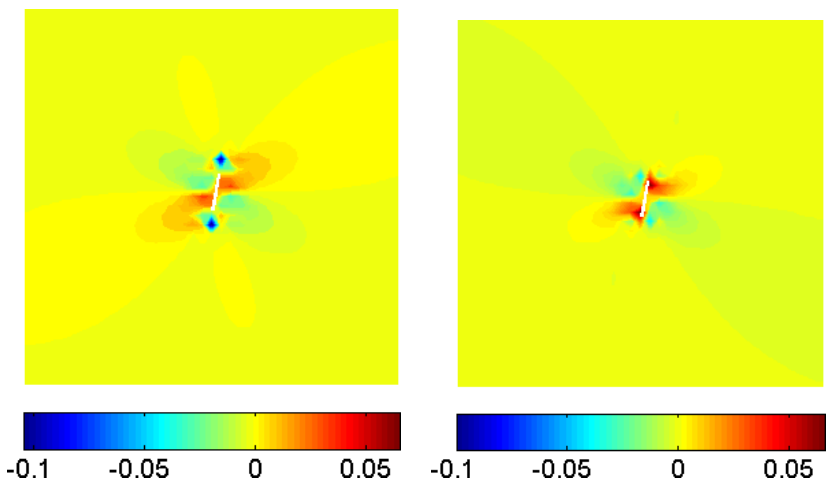

$-0.1-0.05$

(c) $\sigma_{11}$ in $\mathrm{MPa}$, Standard XFEM

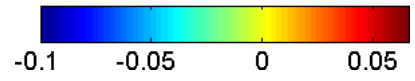

(d) $\sigma_{11}$ in MPa, Present XFEM
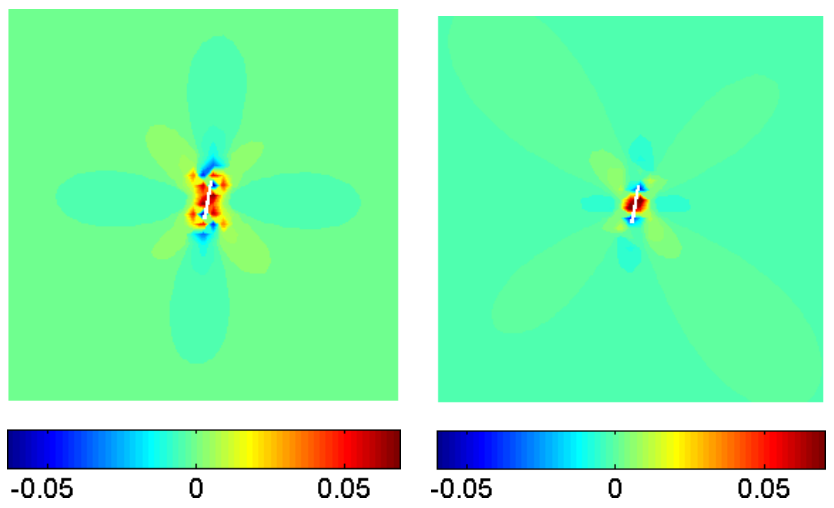

(e) $\sigma_{12}$ in $\mathrm{MPa}$, Standard

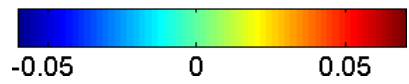
XFEM

Figure 21: CCT: contour plots of $\sigma_{22}, \sigma_{11}$, and $\sigma_{12}$ for $\alpha=80^{\circ}, 2 a=W / 50$, and 199x199 finite elements. 